OPEN ACCESS

Edited by:

Soumen Basak,

National Institute of Immunology (NII),

India

Reviewed by:

Payel Roy,

La Jolla Institute for Immunology (LJI),

United States

Sunil Kumar Raghav,

Institute of Life Sciences (ILS), India

*Correspondence:

Qinmiao Sun

qinmiaosun@ioz.ac.cn

${ }^{+}$These authors have contributed equally to this work

Specialty section:

This article was submitted to

Signaling,

a section of the journal

Frontiers in Cell and Developmental

Biology

Received: 17 May 2021

Accepted: 26 July 2021

Published: 19 August 2021

Citation:

Liu E, Sun J, Yang J, Li L, Yang Q

Zeng J, Zhang J, Chen D and Sun Q (2021) ZDHHC11 Positively Regulates $N F-\kappa B$ Activation by Enhancing

TRAF6 Oligomerization.

Front. Cell Dev. Biol. 9:710967. doi: 10.3389/fcell.2021.710967

\section{ZDHHC11 Positively Regulates NF-KB Activation by Enhancing TRAF6 Oligomerization}

\author{
Enping Liu'1,2,3†, Jiawei Sun 1,2,3+, Jing Yang ${ }^{1,4}$, Lin Li ${ }^{1,2}$, Qili Yang ${ }^{1,2,3}$, Jiuqin Zeng ${ }^{1,2,3}$, \\ Jiayu Zhang ${ }^{1,2,3}$, Dahua Chen ${ }^{5}$ and Qinmiao Sun ${ }^{1,2,3 *}$
}

'State Key Laboratory of Membrane Biology, Institute of Zoology, Chinese Academy of Sciences, Beijing, China, ${ }^{2}$ Institute of Stem Cells and Regeneration, Chinese Academy of Sciences, Beijing, China, ${ }^{3}$ School of Life Sciences, University of Chinese Academy of Sciences, Beijing, China, ${ }^{4}$ Institute of Physical Science and Information Technology, Anhui University, Hefei, China, ${ }^{5}$ Institute of Biomedical Research, Yunnan University, Kunming, China

Tumor necrosis factor receptor-associated factor 6 (TRAF6) is a RING domain ubiquitin ligase that plays an important role in nuclear factor- $\mathrm{kB}(\mathrm{NF}-\mathrm{\kappa} B)$ signaling by regulating activation of the TAK1 and IKK complexes. However, the molecular mechanisms that regulate TRAF6 E3 activity remain unclear. Here, we found that ZDHHC11, a member of the DHHC palmitoyl transferase family, functions as a positive modulator in NF-kB signaling. ZDHHC11 overexpression activated NF-kB, whereas ZDHHC11 deficiency impaired NF-kB activity stimulated by IL-1 $\beta$, LPS, and DNA virus infection. Furthermore, Zdhhc11 knockout mice had a lower level of serum IL6 upon treatment with LPS and D-galactosamine or HSV-1 infection than control mice. Mechanistically, ZDHHC11 interacted with TRAF6 and then enhanced TRAF6 oligomerization, which increased E3 activity of TRAF6 for synthesis of K63-linked ubiquitination chains. Collectively, our study indicates that ZDHHC11 positively regulates NF- $\mathrm{KB}$ signaling by promoting TRAF6 oligomerization and ligase activity, subsequently activating TAK1 and IKK complexes.

Keywords: ZDHHC11, NF-кB, TRAF6, oligomerization, inflammation

\section{INTRODUCTION}

The nuclear factor- $\mathrm{\kappa} \mathrm{B}(\mathrm{NF}-\mathrm{\kappa} \mathrm{B})$ transcription factor not only plays important roles in mediating immune responses, cell proliferation and death, but also is critical for inflammatory responses (Taniguchi and Karin, 2018). It can be activated by stimulation of various receptors such as Tolllike receptors (TLRs), IL-1 receptor (IL-1R), TNFR1, RIG-I-like receptors, and cGAS (Yoneyama et al., 2004; Verstrepen et al., 2008; Hopfner and Hornung, 2020). IL-1//IL-1R and LPS/TLR4mediated NF- $\kappa$ B signaling share a similar signaling pathway. Receptor IL-1R as well as TLR4 recruit the adaptor protein MyD88 after binding to ligand IL-1 or LPS, respectively, then MyD88 forms complex with IL-1R-associated kinases (IRAKs), including IRAK1, IRAK2, and IRAK4, and Tumor necrosis factor receptor-associated factor 6 (TRAF6; Janssens and Beyaert, 2002). Once the MyD88 complex is activated, TRAF6 servers as an E3 ubiquitin ligase to catalyze the synthesis of K63-linked polyubiquitin chains conjugated to itself or other proteins or as free ubiquitin chains (Shi and Sun, 2018). K63-linked polyubiquitin chains bind to TAB2 and NEMO to recruit TAK1-TAB1-TAB2 or TAB3 and the IKB kinase IKK $\alpha$-IKK $\beta$-NEMO complex, respectively, which facilitates TAK1 and IKK activation (Kanayama et al., 2004). Activated TAK1 triggers IKK complex activation and then 
the IKK complex phosphorylates I $\mathrm{KB}$ protein that binds to $\mathrm{NF}-\kappa \mathrm{B}$ in the cytoplasm of resting cells, which results in its ubiquitination and degradation. NF- $\kappa \mathrm{B}$ is released from association with $\mathrm{I} \kappa \mathrm{B}$, and then translocates to the nucleus to trigger transcription of proinflammatory cytokines such as tumor necrosis factor (TNF $\alpha$ ), IL-1 $\beta$, IL-8, and IL-6 (Adhikari et al., 2007; Mulero et al., 2019).

Tumor necrosis factor receptor-associated factor 6 belongs to the tumor necrosis factor receptor-associated factor (TRAF) family, and plays important roles in activation of NF- $\kappa \mathrm{B}$ signaling by IL-1 $\beta$ and LPS (Cao et al., 1996; Lomaga et al., 1999). TRAF6 is also involved in regulating the activation of NF- $\kappa$ B signaling induced by virus infection. Previous studies showed that while TRAF6 plays a redundant role with TRAF2 and TRAF5 in activating NF- $\kappa$ B induced by RNA virus infection (Liu et al., 2013), TRAF6 is critical for the activation of NF- $\kappa \mathrm{B}$ induced by DNA virus infection (Abe and Barber, 2014). In NF- $\mathrm{B}$ signaling, TRAF6 acts as an E3 ubiquitin ligase together with the Ub-conjugating enzyme (E2) complex Ubc13-Uev1A to synthesize polyubiquitin chains linked through Lys-63 (K63) of Ub (Deng et al., 2000). The polyubiquitin chains of K63-linked ubiquitin bind to TAB2 and NEMO, which activates the TAK1 and IKK complex, respectively, Deng et al. (2000), Kanayama et al. (2004), and Wu et al. (2006). TRAF6 harbors an N-terminal RING finger domain, followed by $\mathrm{Zn}$ Finger domains and the C-terminal TRAF domain (Xie, 2013). The N-terminal RING and ZF1 domains constitute the minimal unit to catalyze K63-linked polyubiquitin chain synthesis in vitro, and the C-terminal TRAF-C domain facilitates its oligomerization and association with receptors and adaptor proteins (Ye et al., 2002; Fu et al., 2018). TRAF6 oligomerization is important for its E3 activity (Yin et al., 2009; Fu et al., 2018). A previous study has shown that TIFA promotes TRAF6 oligomerization and ubiquitination (Ea et al., 2004). Although previous studies have made significant progress in delineating the functions of TRAF6, the mechanism of TRAF6 regulation remains unclear.

Protein S-palmitoylation is a reversible post-translational modification that is dynamically controlled by palmitoyl acyl transferases and palmitoyl thioesterase (Yount et al., 2013; Zaballa and van der Goot, 2018). Palmitoylated proteins are involved in regulating numerous protein properties including trafficking, localization, stability, activity, and association with other proteins (Daniotti et al., 2017; Ko and Dixon, 2018). The majority of protein palmitoylation is catalyzed by the family of palmitoyl acyl transferases that have a zinc finger DHHC (ZDHHC) domain required for palmitoyl transfer activity (Mitchell et al., 2006). There are 23 members of the diverse DHHC protein family in humans, which include ZDHHC1ZDHHC24 (ZDHHC10 is omitted) in humans (Korycka et al., 2012; Lemonidis et al., 2015). Previous studies have shown that ZDHHC11 is an ER-associated protein and that its aberrant expression is related to the development of some cancers (Yamamoto et al., 2007; Kang et al., 2008; Gorleku et al., 2011; Wu et al., 2013; Dzikiewicz-Krawczyk et al., 2017). Recently, ZDHHC11 was found to regulate innate immune responses against DNA virus infection by mediating the MITA-IRF3 association (Liu et al., 2018). However, whether ZDHHC11 is involved in inflammatory pathways remains unclear.

In the present study, we identified ZDHHC11 as a positive modulator in NF- $\kappa \mathrm{B}$ signaling, and found that ZDHHC11 was involved in regulating the activity of NF- $\kappa \mathrm{B}$ stimulated by IL-1 $\beta$, LPS, and DNA virus infection. $Z d h h c 11^{-/-}$mice exhibited a lower level of serum IL-6 upon treatment with LPS and D-galactosamine or HSV-1 infection than control mice. Moreover, we demonstrated that ZDHHC11 associated with TRAF6 and then increased TRAF6 oligomerization, which enhanced TRAF6 E3 activity to synthesize K63-linked ubiquitination chains. Taken together, we found that ZDHHC11 increases oligomerization and E3 activity of TRAF6, which leads to activation of TAK1 and IKK, and then positively modulates NF- $\kappa$ B signaling.

\section{RESULTS}

\section{ZDHHC11 Positively Regulates IL-1 $\beta$-Induced NF- $\kappa$ B Activation}

To determine whether ZDHHC family members play a role in innate immune signaling, HEK293T cells were cotransfected 16 independent cDNA expression plasmids that encoded members of the ZDHHC family with a luciferase gene under the control of the IFN $\beta$ promoter (IFN $\beta$-Luc), which contains NF- $\kappa$ B and IRF3 binding sites. As a result, we found that ZDHHC11 significantly induced IFN $\beta$ activity compared with other ZDHHC family members (Supplementary Figure 1). Because IFN- $\beta$ induction requires the coordinated action of both IRF3 and NF- $\kappa$, we next investigated how ZDHHC11 activated the IFN $\beta$ promoter. We employed an NF-кB luciferase reporter and IFN-stimulated response element (ISRE) luciferase reporter that is activated by IRF3. Reporter assays showed that ZDHHC11 overexpression activated IFN $\beta$ and NF- $\kappa B$, but not the ISRE reporter, in a dose-dependent manner in HEK293T cells (Figures 1AC). Consistently, quantitative reverse transcription-PCR (qRTPCR) assays demonstrated that ZDHHC11 overexpression in HEK293T cells increased the mRNA levels of NF- $\kappa$ B downstream genes $T N F \alpha$ and IL8, but not IRF3-dependent genes, such as IFIT1 (Figures 1D-G). Similarly, ZDHHC11 overexpression increased the mRNA levels of TNF $\alpha, I L 6$, and IL8 in HeLa cells (Supplementary Figures 2A-D). Because activation of NF- $\kappa$ B signaling induces p65 phosphorylation and nuclear translocation, we next examined whether ZDHHC11 overexpression affected these characteristics. Western blotting results showed that ZDHHC11 overexpression increased the level of phosphorylated p65 in a dose-dependent manner (Figure 1H and Supplementary Figure 2E) and p65 nuclear translocation was induced when ZDHHC11 was overexpressed in HEK293T cells (Figure 1I). These data suggest that ZDHHC11 overexpression specifically activates NF- $\kappa$ B signaling.

Proinflammatory cytokine interleukin-1 $\beta$ (IL-1 $\beta$ ) and TNF $\alpha$ trigger NF- $\kappa \mathrm{B}$ activation, next, we tried to determinate whether ZDHHC11 played a role in regulating IL-1 $\beta$ - and $\mathrm{TNF} \alpha-$ mediated NF- $\kappa \mathrm{B}$ activation. qRT-PCR assays indicated that ZDHHC11 overexpression significantly increased the IL8 mRNA 
A

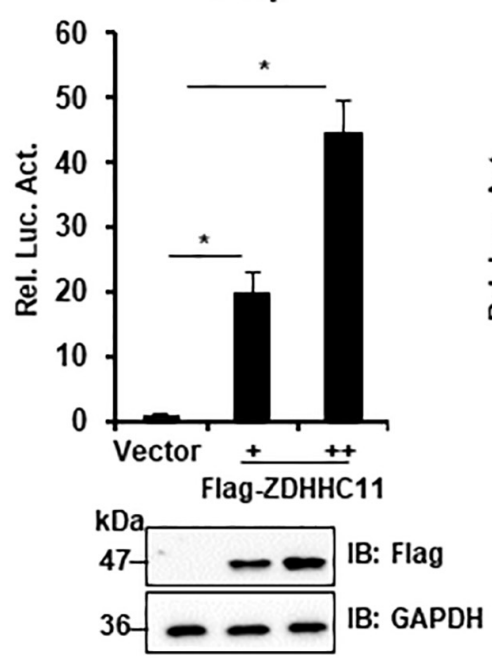

B

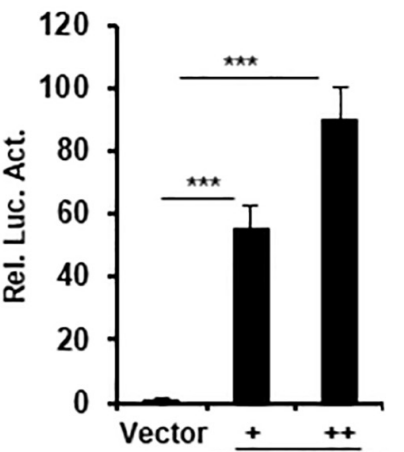

Flag-ZDHHC 11

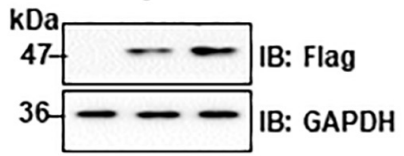

C
ISRE
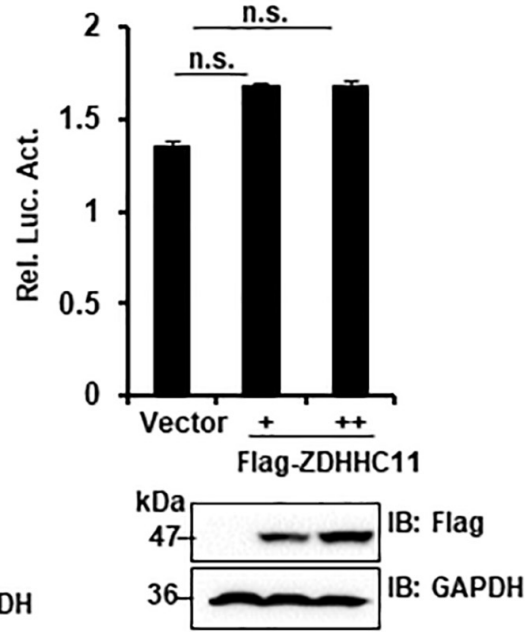

D

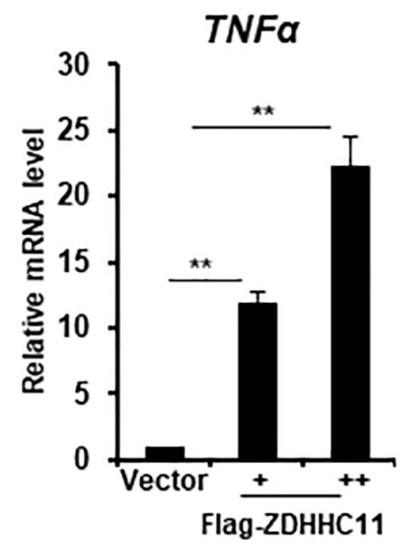

G

Flag-ZDHHC11 $-\quad+{ }^{++} \mathrm{kDa}$

IB: Flag

IB: GAPDH

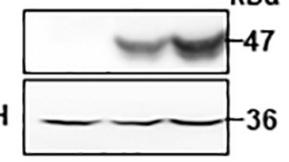

H

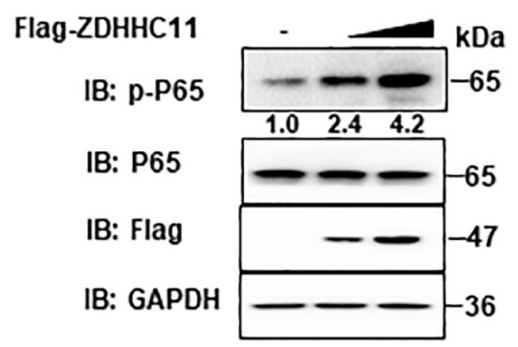

E

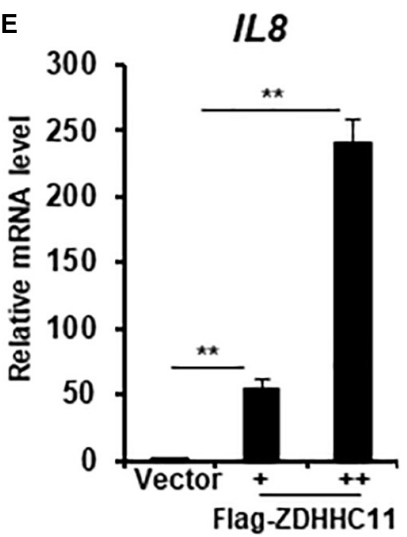

I

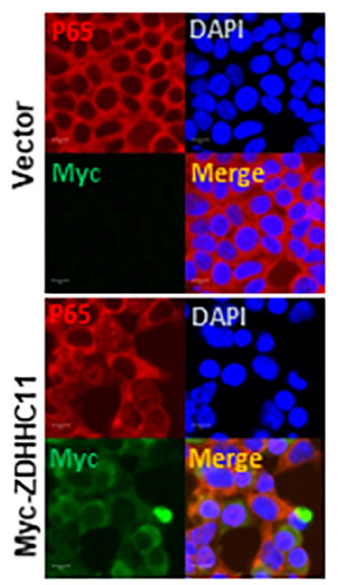

$\mathbf{F}$

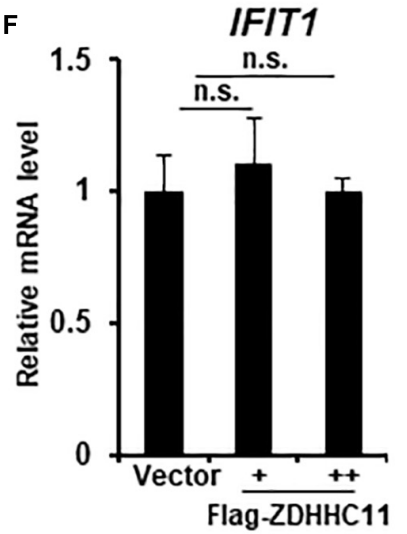

J

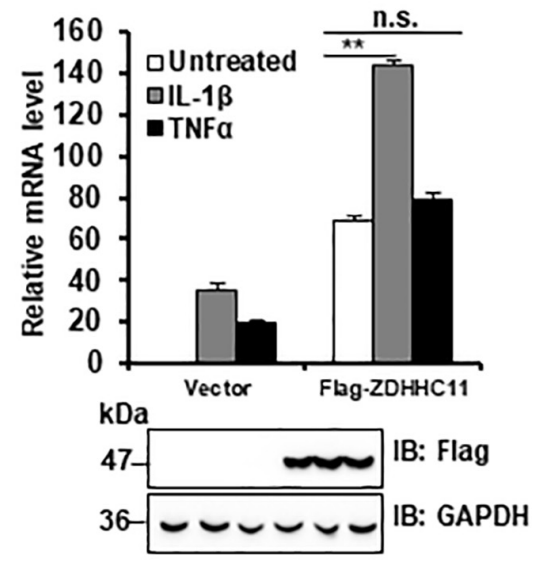

FIGURE 1 | ZDHHC11 overexpression activates NF-kB signaling. (A-C) HEK293T cells were cotransfected with an empty vector or different doses of ZDHHC11 (50 and $100 \mathrm{ng}$ ) expression plasmids and luciferase reporter plasmids IFN- $\beta$-Luc (100 ng; A), NF-кB-Luc (20 ng; B), or ISRE-Luc (100 ng; C). After 24 h, the cells were harvested for luciferase reporter assay (upper panel) and immunoblot assay (lower panels). (D-G) HEK293T cells were transfected with empty vector or different 


\section{FIGURE 1 | Continued}

amount of ZDHHC11 expression plasmids (50 and $100 \mathrm{ng}$ ). After $24 \mathrm{~h}$, the cells were harvested to isolate RNA or protein, followed by qRT-PCR analysis to measure the mRNA levels of TNF $\alpha$ (D), IL8 (E), and IFIT1 (F) or immunoblotting to detect ZDHHC11 protein expression (G), respectively. (H) HEK293T cells were transfected with different doses of ZDHHC11 expression plasmids (50 and $100 \mathrm{ng}$ ) or empty vector. After $24 \mathrm{~h}$, the cells were harvested for immunoblot assay with the corresponding antibodies. (I) HEK293T cells were transfected with Myc-tagged ZDHHC11 expression plasmids (200 ng) or empty vector. After 24 h, the cells were fixed, stained with the indicated antibodies. The images were taken by confocal microscopy. Scale bars, $10 \mu \mathrm{m}$. (J) HEK293 C6 cells were transfected with indicated plasmids (200 ng). After $24 \mathrm{~h}$, the cells were untreated or treated by IL-1 $\beta$ (10 ng/ml) or TNF $\alpha$ (10 ng/ml) for $10 \mathrm{~h}$, followed by qRT-PCR analysis (upper panel) or immunoblot assay (lower panels). Data shown in (A-F,J) are representative of three independent experiments (mean \pm SD of duplicate experiments). ${ }^{\star} P<0.05$; ${ }^{\star \star} P<0.01$; ${ }^{\star \star} P<0.001$; n.s. not significant versus the control groups; and Student's $t$-test. All blots are representative of three independent experiments.

level induced by IL-1 $\beta$, but not TNF $\alpha$, in HEK293 C6 cells that ectopically express IL-1R (Figure 1J). These data suggest that ZDHHC11 specifically enhances NF-кB activation triggered by IL- $1 \beta$.

\section{ZDHHC11 Knockdown Decreases IL-1 $\beta$-Induced NF- $\kappa$ B Activation}

To further determinate the biological functions of endogenous ZDHHC11 in modulating NF- $\mathrm{BB}$ activation, we employed two lentivirus-delivered shRNAs that specifically targeted nonoverlapping regions of the coding region of human $Z D H H C 11$ and evaluated whether knockdown of $Z D H H C 11$ affected NF-кB signaling in HEK293 C6 cells. As shown in Figures 2A-C, both shRNA-ZDHHC11-1/2 efficiently reduced the level of ZDHHC11 mRNA and knockdown of ZDHHC11 significantly reduced transcriptional levels of IL8 and TNF $\alpha$ after IL- $1 \beta$ stimulation in a time-independent manner compared with control cells. Consistently, the levels of phosphorylated TAK1, IKK $\alpha / \beta$, and p65 were decreased in ZDHHC11 knockdown cells after IL-1 $\beta$ stimulation (Figures 2D,E and Supplementary Figure 3A). To determine the specific role of ZDHHC11, we conducted rescue experiments, and observed that restored expression of ZDHHC11 reversed the reduced levels of phosphorylated TAK1 and IL8 mRNA induced by IL-1 $\beta$ stimulation in ZDHHC11 knockdown cells (Figures 2F,G). Additionally, we performed a similar knockdown assay in HeLa cells and obtained similar qRT-PCR results as those in HEK293 C6 cells (Supplementary Figures 3BD). Western blotting also indicated that knockdown of $Z D H H C 11$ reduced the levels of phosphorylated TAK1, IKK $\alpha / \beta$, and I $\mathrm{B} \alpha$ stimulated by IL-1 $\beta$ (Supplementary Figures 3E,F). Taken together, these results support the notion that ZDHHC11 positively modulates the NF- $\mathrm{B}$ signaling.

\section{Zdhhc11 Deficiency Reduces NF-кB Activation Stimulated by IL-1 $\beta$ and LPS Treatments as Well as DNA Virus Infection}

To further elucidate the physiological roles of ZDHHC11 in NF$\kappa \mathrm{B}$ activation, we employed $Z d h h c 11$-deficient mice from Jackson Lab. We generated mouse embryonic fibroblasts (MEFs) from $Z d h h c 11^{+/+}$and $Z d h h c 11^{-/-} 13.5$-day-old embryos by breeding heterozygote mutants (Supplementary Figure 4A) and then examined the effect of $Z d h h c 11$ deficiency on NF-кB signaling. qRT-PCR showed that $Z d h h c 11$ knockout significantly reduced the mRNA levels of Il6 and $\operatorname{Tn} f \alpha$ after stimulation by IL-1 $\beta$ compared with WT controls (Figures 3A,B). Consistently, an enzyme linked immunosorbent assay (ELISA) showed that IL6 protein induced by IL-1 $\beta$ was lower in $Z d h h c 11^{-/-}$MEFs than in $Z d h h c 11^{+/+}$control cells (Figure 3C). Additionally, western blotting indicated that $Z d h h c 11$ knockout in MEFs reduced the levels of phosphorylated TAK1, I $\mathrm{B} \alpha$, and I $\mathrm{B} \alpha$ degradation after IL-1 $\beta$ stimulation (Figure 3D). Next, we determined the effect of $Z$ dhhc11 deficiency on NF- $\mathrm{B}$ signaling in bone marrow-derived macrophages (BMDMs). qRT-PCR assays demonstrated that the mRNA levels of $I l 6$ and $I l-1 \beta$ were significantly attenuated in Zdhhc11 $1^{-/-}$BMDMs compared with $Z d h h c 11^{+/+}$cells after IL$1 \beta$ stimulation (Supplementary Figures 4B,C). These findings indicate that ZDHHC11 plays a critical role in IL- $1 \beta$-triggered $\mathrm{NF}-\kappa \mathrm{B}$ activation in MEFs and macrophages.

Considering that both LPS and IL- $1 \beta$ trigger NF- $\kappa$ B activation and share similar intracellular signaling pathways (Narayanan and Park, 2015), we next determined whether ZDHHC11 is involved in LPS-induced NF- $\kappa \mathrm{B}$ activation. qRT-PCR assays indicated that the mRNA levels of Il6 and Tnf $\alpha$ after LPS stimulation were significantly lower in $Z d h h c 11^{-/-}$MEFs (Supplementary Figures 4D,E) and BMDMs (Figures 3E,F) compared with their $Z d h h c 11^{+/+}$counterparts. ELISA results also showed that Zdhhc11 deficiency reduced the production of IL6 and TNF $\alpha$ induced by LPS stimulation in BMDMs (Supplementary Figures 4F,G). Consistently, the levels of phosphorylated TAK1, IKK $\alpha / \beta$, and $\mathrm{p} 65$ in $Z d h h c 11^{-/-}$BMDMs were lower than those in $Z d h h c 11^{+/+}$BMDMs (Figure 3G). These findings indicate that ZDHHC11 also plays a critical role in LPS-triggered NF- $\kappa$ B activation.

Next, we examined whether ZDHHC11 was involved in NF$\kappa \mathrm{B}$ activation induced by virus infection. As shown in Figure $3 \mathbf{H}$, Zdhhc11 deficiency in BMDMs decreased the mRNA level of Il6 induced by infection with herpes simplex virus (HSV-1), but it had no effect on the expression of Il6 mRNA stimulated by Sendai virus, a kind of RNA virus. Consistent with the results in BMDMs, mRNA levels of Il6 and Tnf $\alpha$ were also lower in Zdhhc11-/- MEFs than wild-type cells upon HSV-1 infection (Supplementary Figures 4H,I). Additionally, we observed that ZDHHC11 deficiency in MEFs reduced the mRNA levels of Ifnb1 and Ifit1, one IRF3-dependent gene, induced by HSV-1, which was consistent with the previous study showing ZDHHC11 modulates the innate immune response to DNA virus infection (Supplementary Figures 4J,K). Collectively, these data suggest that ZDHHC11 also played a positive role in regulating NF- $\kappa \mathrm{B}$ signaling triggered by DNA virus infection.

To determinate whether ZDHHC11 is involved in NF- $\mathrm{B}$ signaling in vivo, we first treated $Z d h h c 11^{+/+}$and $Z d h h c 11^{-/-}$ mice with LPS and D-galactosamine by intraperitoneal injection 

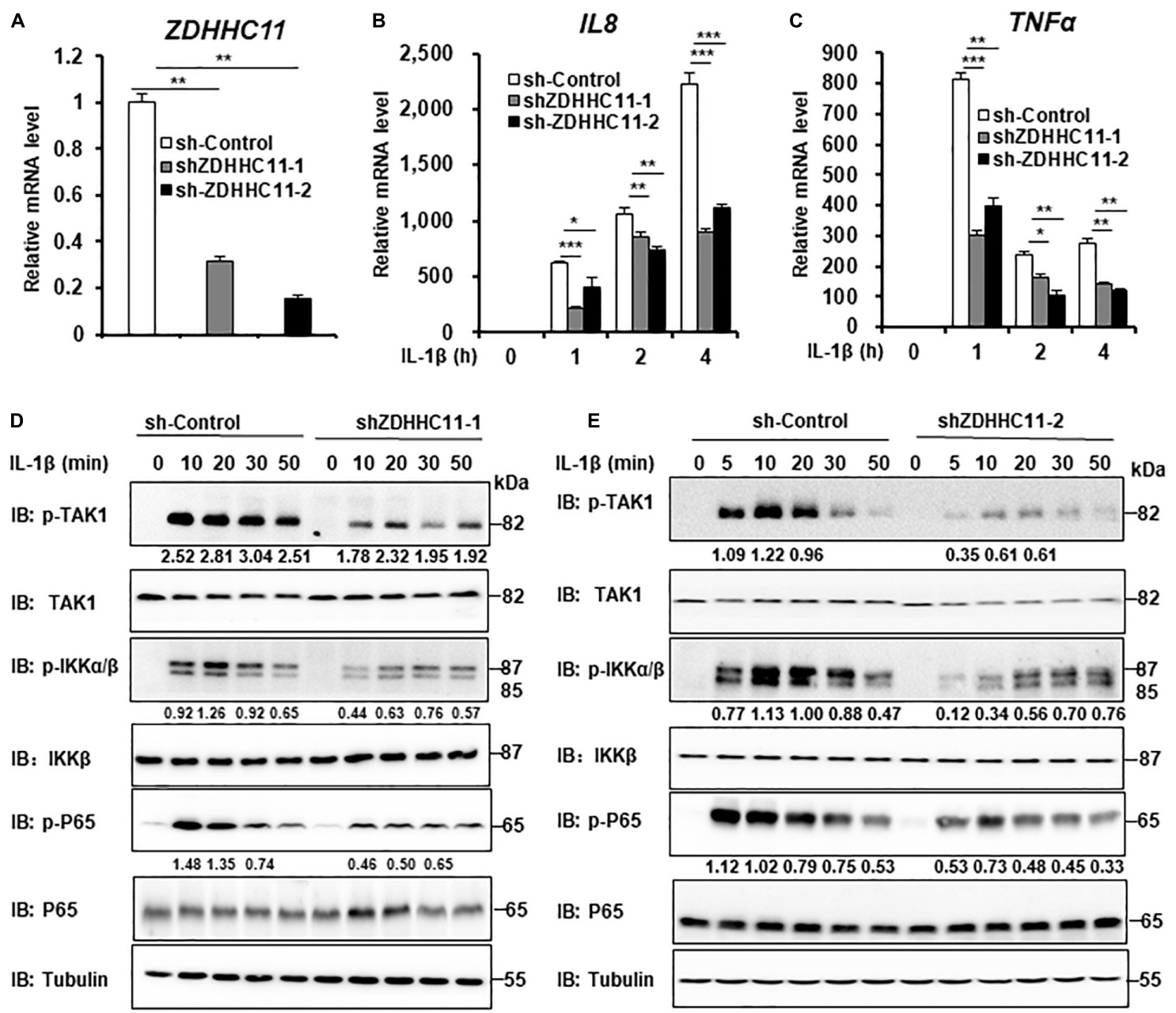

IB: TAK1

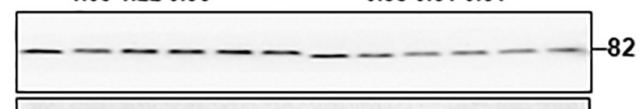

IB: $\mathrm{p}-\mathrm{IKKa} / \beta$

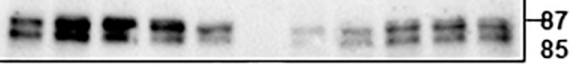

$\begin{array}{lllllllll}0.77 & 1.131 .00 & 0.88 & 0.47 & 0.12 & 0.34 & 0.56 & 0.70 & 0.76\end{array}$

IB: IKK $\beta$

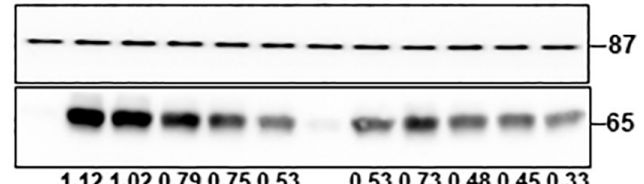

IB: p-P65

$1.121 .020 .790 .750 .53 \quad 0.530 .730 .480 .450 .33$

IB: P65

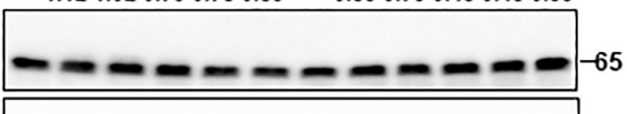

IB: Tubulin

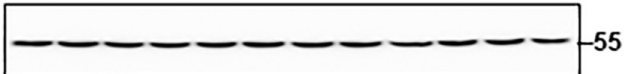

$\mathbf{F}$

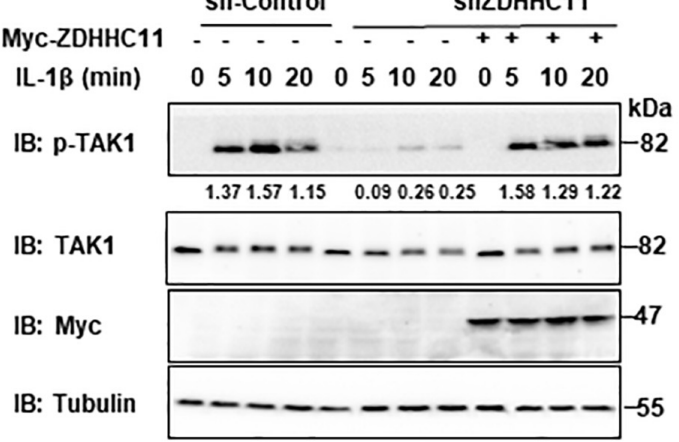

G

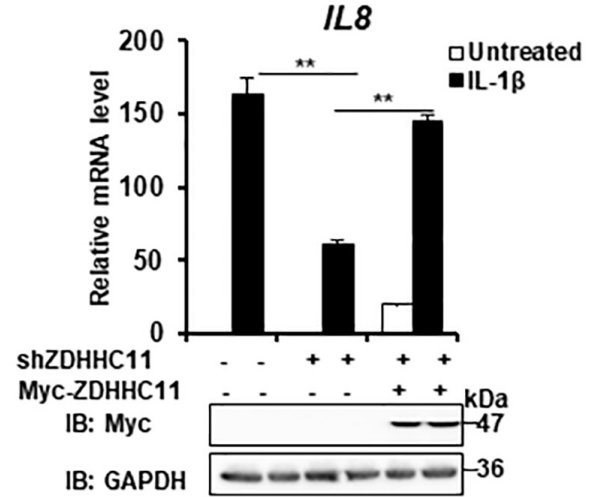

FIGURE 2 | ZDHHC11 knockdown attenuates IL-1 $\beta$-induced NF-KB activation. (A-C) HEK293 C6 cells were infected with lentivirus expressing shRNA that targeted different coding regions of human ZDHHC11 (shZDHHC11-1 and shZDHHC11-2) or an empty vector for $48 \mathrm{~h}$ and then unstimulated or stimulated with IL-1 $\beta$ $(10 \mathrm{ng} / \mathrm{ml})$ for the indicated times. qRT-PCR assays were performed to examine the mRNA levels of ZDHHC11 (A), IL8 (B), and TNF $\alpha$ (C). (D,E) Infection was performed as described in (A). The cells were unstimulated or stimulated with IL-1 $\beta(10 \mathrm{ng} / \mathrm{ml})$ for the time as indicated. Cells were harvested to perform immunoblot assay. (F) HEK293 C6 cells were infected with lentivirus expressing shRNA that targeted ZDHHC11 or an empty vector for $48 \mathrm{~h}$ and then transfected with a ZDHHC11 expression plasmid (500 ng) or empty vector as indicated. After $24 \mathrm{~h}$ of transfection, the cells were simulated with IL-1 $\beta$ ( $10 \mathrm{ng} / \mathrm{ml})$ for the indicated times, followed by immunoblot analysis. (G) HEK293 C6 cells were infected and transfected as described in (F) and then treated with IL-1 $1 \beta$ for $3 \mathrm{~h}$, followed by qRT-PCR analysis. Data shown in $(\mathbf{A}-\mathbf{C}, \mathbf{G})$ are representative of three independent experiments (mean \pm SD of duplicate experiments). ${ }^{\star} P<0.05 ;{ }^{* \star} P<0.01 ;{ }^{\star \star \star} P<0.001$ versus the control groups; and Student's $t$-test. All blots are representative of three independent experiments. 

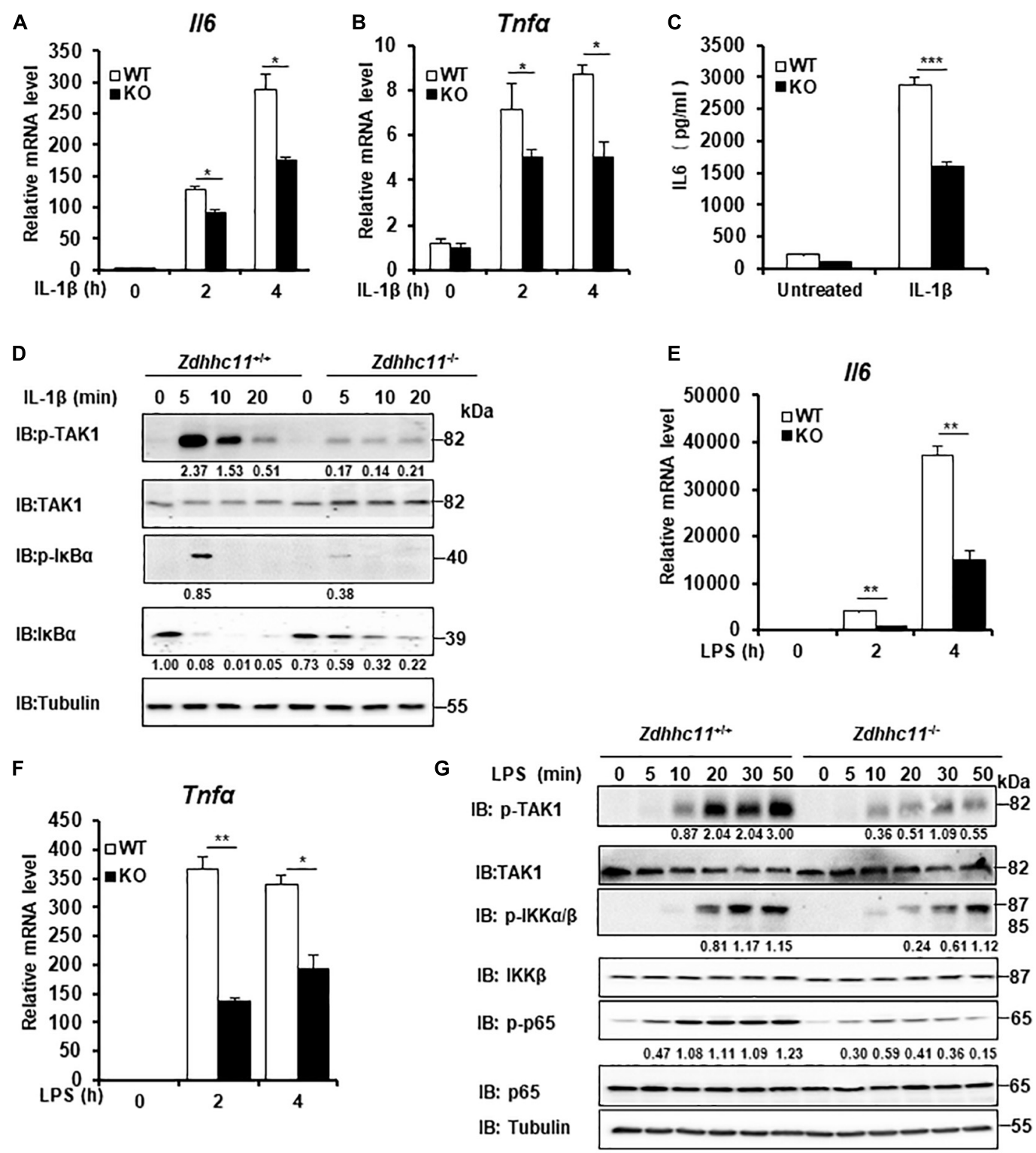

G
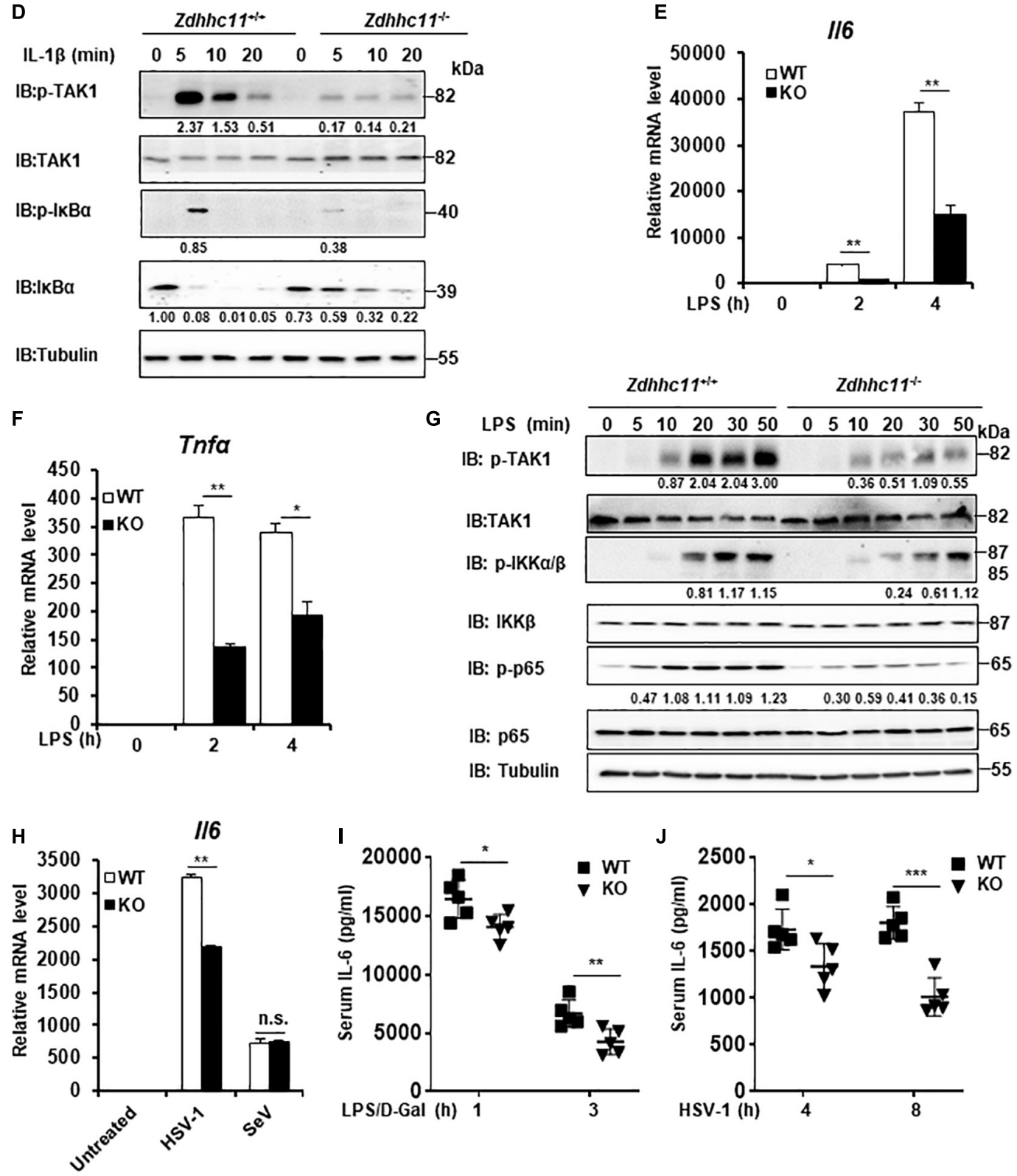

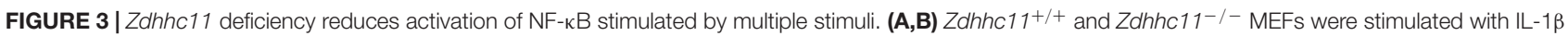
$\left(20 \mathrm{ng} / \mathrm{ml}\right.$ ) for the time as indicated, then qRT-PCR assay was performed to measure transcriptional levels of $/ / 6$ (A) and Tnfa (B). (C) Zdhhc11 ${ }^{+/+}$and Zdhhc1 $11^{-/-}$

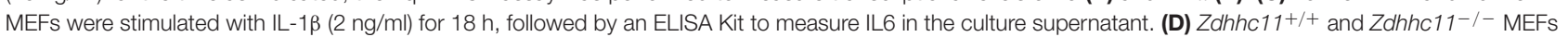

(Continued) 


\section{FIGURE 3 | Continued}

were treated with IL-1 $\beta$ (20 ng/ml) for the indicated times, followed by immunoblot analysis. (E,F) Zdhhc11 ${ }^{+/+}$and Zdhhc11-/- BMDMs were treated with LPS (20 ng/ml) for 0, 2, or $4 \mathrm{~h}$ and then analyzed by qRT-PCR assays to examine transcriptional levels of $/ / 6$ (E) and Tnf $\alpha$ (F). (G) Zdhhc11 ${ }^{+/+}$and Zdhhc11-/- BMDMs were treated with LPS $(30 \mathrm{ng} / \mathrm{ml})$ for the indicated times. The cell extracts were harvested for immunoblot analysis. (H) Zdhhc11 ${ }^{+/+}$and $Z d h h c 11^{-/-}$BMDMs were infected with HSV-1 (5MOI) or SeV (10HA) for $6 \mathrm{~h}$, then qRT-PCR assays were performed to measure the mRNA level of //6. (I) Zdhhc11+/+ and Zdhhc11-/- mice $(n=5)$ were treated with LPS and D-galactosamine via intraperitoneal injection. Sera were collected at 1 and $3 \mathrm{~h}$ after injection to measure IL-6 levels by an ELISA

Kit. (J) Zdhhc $11^{+/+}$and Zdhhc11-/- mice $(n=5)$ were infected with HSV-1 via intravenous injection at $2 \times 10^{7}$ PFU per mouse. Sera were collected at 4 and $8 \mathrm{~h}$ after infection to measure IL-6 by an ELISA kit. Data shown in (A-C,E,F,H) are representative of three independent experiments (mean \pm SD of duplicate experiments). ${ }^{\star} P<0.05$; ${ }^{\star \star} P<0.01$; ${ }^{\star \star \star} P<0.001$; n.s. not significant versus the control groups; and Student's $t$-test. All blots are representative of three independent experiments.

and then measured IL- 6 in their sera by ELISA. As shown in Figure 3I, the level of IL-6 protein in $Z d h h c 11^{-/-}$mice was significantly lower than that in control mice. Next, we infected Zdhhc11 $1^{+/+}$and $Z d h h c 11^{-/-}$mice with HSV-1 by intravenous injection and measured IL-6 in sera. As a result, Zdhhc11 knockout mice also showed a significantly reduced level of IL-6 compared with control mice (Figure 3J). These results provide evidence that ZDHHC11 plays an important role in regulating NF- $\mathrm{B}$ signaling in vivo.

\section{ZDHHC11 Targets TRAF6 to Regulate NF- $\kappa B$ Signaling}

Our results described above demonstrated that ZDHHC11 was involved in modulating NF- $\kappa$ B activation stimulated by IL$1 \beta$, LPS, and DNA virus infection. Next, we tried to explore the molecular mechanism by which ZDHHC11 regulates NF$\kappa \mathrm{B}$ signaling. To identify ZDHHC11-targeted proteins, we first conducted co-immunoprecipitation (Co-IP) to test whether ZDHHC11 interacted with known components of the NF- $\kappa$ B pathway. We cotransfected ZDHHC11 with TAK1, TRAF6, IKK $\alpha$, IKK $\beta$, NEMO, and p65 into HEK293T cells and found that overexpressed ZDHHC11 strongly associated with TRAF6 and weakly associated with TAK1 and IKK $\alpha$, whereas no interaction was detected between ZDHHC11 and IKK $\beta$, NEMO, or p65 (Figure 4A). Because TRAF2, TRAF3, and TRAF5 have similar structures to TRAF6 and all play important roles in NF- $\kappa$ B signaling (Nakano et al., 1996; Park et al., 1999; Park, 2018), we next investigated whether ZDHHC11 also associated with these TRAF family members. Co-IP indicated that ZDHHC11 pulldowned TRAF6 but not TRAF2, TRAF3, or TRAF5 (Figure 4B). To further investigate the specificity of the interaction between ZDHHC11 and TRAF6, we evaluated whether Flag-tagged ZDHHC11 interacted with endogenous components of NF$\kappa \mathrm{B}$ pathway. As shown in Figure 4C, ectopic expression of ZDHHC11 was strongly associated with endogenous TRAF6, but not other components. Consistently, ZDHHC11 and TRAF6 were reciprocally co-immunoprecipitated in transfected HEK293T cells (Supplementary Figures 5A,B). These data suggest that ZDHHC11 specifically associated with TRAF6 to regulate $\mathrm{NF}-\kappa \mathrm{B}$ signaling activity.

Next, we determined which domains of ZDHHC11 and TRAF6 were responsible for their interaction. TRAF6 consists of three major domains, an N-terminal RING finger domain, Zn Finger domains, and a C-terminal TRAF domain which is further divided into a TRAF-N domain and a TRAF-C domain
(Yin et al., 2009). We generated several TRAF6-truncated mutants and found that TRAF-C domain of TRAF6 was required for its interaction with $\mathrm{ZDHHC11}$ (Figure 4D and Supplementary Figure 5C). ZDHHC11 mapping indicated that the C-terminal region of ZDHHC11 (198-412 aa) was involved in the association between ZDHHC11 and TRAF6 (Figure 4E). These results indicated that the association between ZDHHC11 and TRAF6 depends on a specific domain.

To further determine whether TRAF6 is a target of ZDHHC11 in NF- $\mathrm{B}$ signaling, we cotransfected TRAF6 with ZDHHC11 or the empty vector together with an NF-кB-Luc reporter into HEK293T cells. The reporter assay showed that ZDHHC11 overexpression synergistically enhanced TRAF6-induced NF$\kappa \mathrm{B}$ activation (Figure 4F). Consistently, qRT-PCR showed that ZDHHC11 overexpression significantly augmented IL8 mRNA expression induced by TRAF6, but not TRAF2 (Figure 4G). Additionally, TRAF6 knockdown by siRNA dramatically reduced the activities of IFN $\beta$ and NF- $\mathrm{B}$ promoters induced by ZDHHC11 overexpression in HEK293T cells (Figures 4H,I). Taken together, these findings further suggest that TRAF6 is the target of ZDHHC11 in regulation of NF- $\mathrm{B}$ B signaling.

\section{ZDHHC11 Enhances TRAF6 E3 Activity by Augmenting TRAF 6 Oligomerization}

Next, we investigated the molecular mechanisms of ZDHHC11, which regulate NF- $\kappa$ B by targeting TRAF6. Because ZDHHC11 is a member of the DHHC palmitoyl acyltransferase family, we examined whether palmitoyl transferase activity is required for its function in NF-кB signaling. In accordance with other members of the DHHC palmitoyl transferase family, specific aspartate-histidine $(\mathrm{DH})$ and cysteine $(\mathrm{C})$ residues in the DHHC domain of ZDHHC members are critical for its palmitoyl transferase activity. Therefore, we constructed several mutants of ZDHHC11, which included ZDHHC11DH/AA (D152A, H153A), ZDHHC11C/S (C155S), and ZDHHC11 $\triangle \mathrm{DHHC}$ (del 152-155 aa), in which DHHC was deleted and then examined their ability to activate NF- $\kappa$ B signaling. Reporter assays demonstrated that these mutants activated NF- $\mathrm{B}$ signaling at similar levels as wild-type ZDHHC11 (Figure 5A). These results suggest that the palmitoyl transferase activity of ZDHHC11 was not required for its NF- $\kappa$ B activation.

Previous studies have demonstrated that TRAF6 oligomerization plays an important role in regulating the activity of NF-кB signaling (Ea et al., 2004; Hu et al., 2017). Therefore, we next examined whether ZDHHC11 modulates 
A

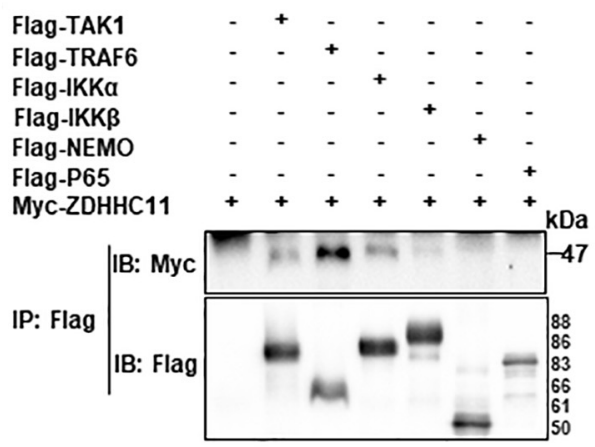

B

WCL |IB: Myc $--\infty-\infty-\infty-47$

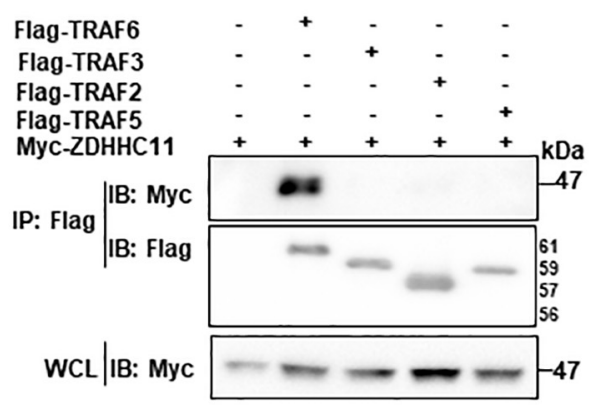

E

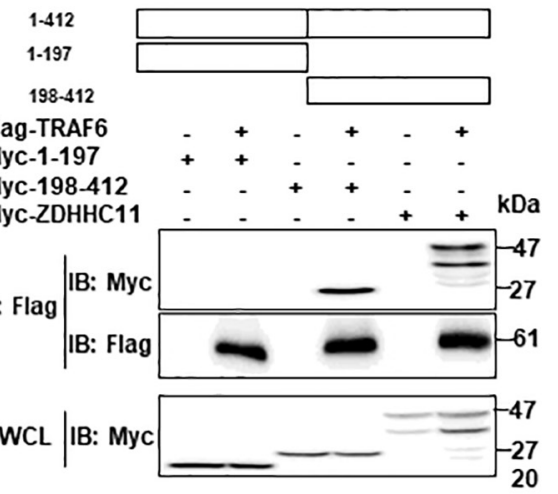

C

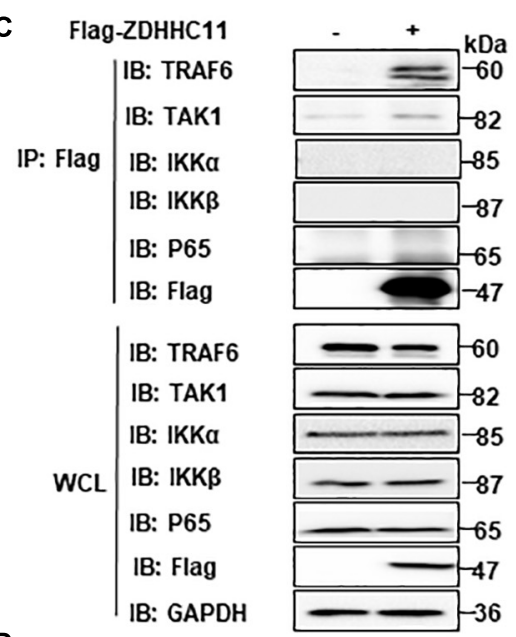

D

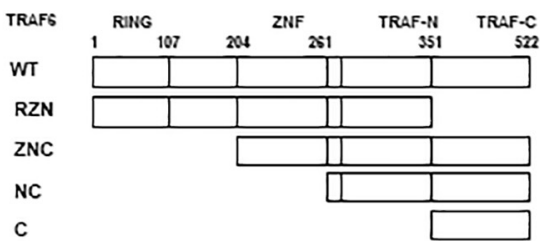

Flag-RZN

Flag-ZNC

Flag-NC

Flag-C

Flag-TRAF6

Myc-ZDHHC11

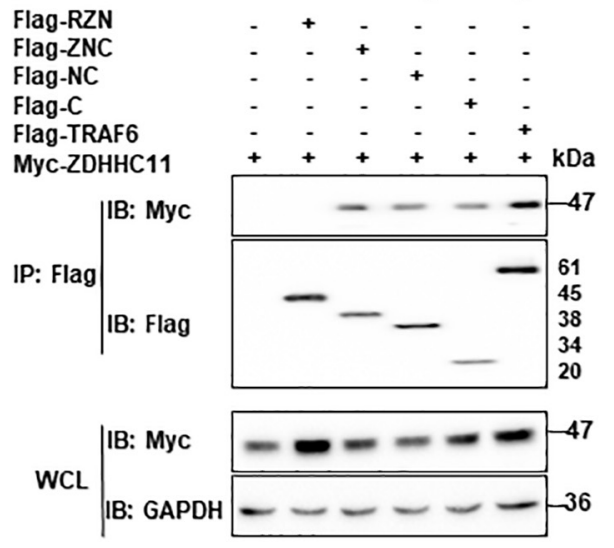

F

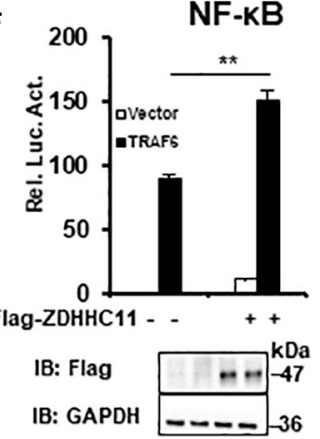

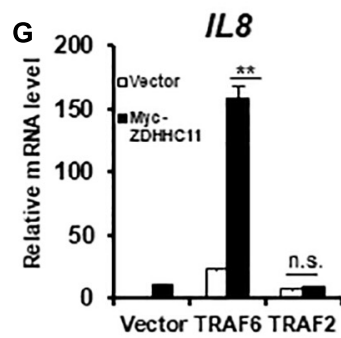

IB: Myc

IB: GAPDH

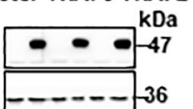

H

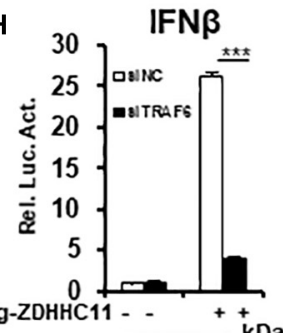

IB: Flag

IB: TRAF6

IB: GAPDH

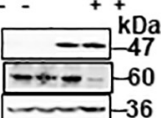

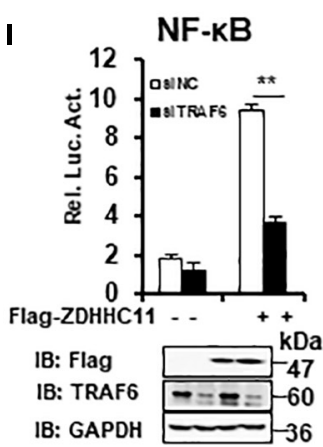

FIGURE 4 | ZDHHC11 targets TRAF6 to modulate NF-KB signaling. (A,B) HEK293T cells were transfected with the indicated expression plasmids, After $24 \mathrm{~h}$ of transfection, co-immunoprecipitation were performed with anti-Flag beads and then followed by immunoblot assay with the corresponding antibodies. (C) HEK293T cells were transfected with an empty vector or Flag-tagged ZDHHC11 $(3 \mu \mathrm{g})$ expression plasmid. $24 \mathrm{~h}$ later, co-immunoprecipitation were performed with anti-Flag beads, followed by immunoblot assay with the corresponding antibodies. (D) HEK293T cells were cotransfected with ZDHHC11 $(2 \mu \mathrm{g})$ and TRAF6 (2 $\mu \mathrm{g})$ or its 


\section{FIGURE 4 | Continued}

truncated mutants, which is illustrated in upper panels. Co-immunoprecipitation were performed with anti-Flag beads and the results of immunoblot assay were showed in lower panels. (E) HEK293T cells were cotransfected with TRAF6 (2 $\mu \mathrm{g})$ or an empty vector together with ZDHHC11 (2 $\mu \mathrm{g})$ or its truncated mutants which is illustrated in upper panels. Co-immunoprecipitation were performed with anti-Flag beads and the results of immunoblot assay were showed in lower panels. (F) HEK293T cells were cotransfected with the Flag-tagged ZDHHC11 (100 ng), HA-tagged TRAF6 (20 ng) and luciferase reporter plasmid NF-kB-Luc (20 ng). $24 \mathrm{~h}$ later, luciferase reporter assays (upper panel) and immunoblot assays (lower panels) were performed. (G) HEK293T cells were cotransfected with the indicated plasmids (ZDHHC11 $100 \mathrm{ng}$, TRAF6 $10 \mathrm{ng}$, and TRAF2 $50 \mathrm{ng}$ ). After $24 \mathrm{~h}$, the cells were collected for qRT-PCR assays to measure the mRNA level of IL8. (H,I) HEK293T cells were transfected with a siRNA that targeted TRAF6 or a non-targeting control (NC). $24 \mathrm{~h}$ later, the cells were cotransfected with an empty vector or ZDHHC11 (100 ng) expression plasmid together with luciferase reporter plasmids IFN $\beta$-Luc (H) or NF-kB-Luc (I). After 24 h of plasmids transfection, the cells were harvested for luciferase reporter assays (upper panel) and immunoblotting (lower panels). Data shown in (F-I) are representative of three independent experiments (mean \pm SD of duplicate experiments). ${ }^{\star \star} P<0.01 ;{ }^{\star \star \star} P<0.001$ significant versus the control groups; Student's $t$-test. All blots are representative of three independent experiments.

TRAF6 oligomerization. Semi-denaturing detergent agarose gel electrophoresis (SDD-AGE) assays were employed to detect TRAF6 oligomerization. As shown in Figure 5B, ZDHHC11 overexpression significantly enhanced TRAF6 oligomerization. Given that the experiments described above indicated C-terminal region of ZDHHC11 (198-412 aa) was important for the association between ZDHHC11 and TRAF6, we then examined whether C-terminal region of $\mathrm{ZDHHC11}$ affects TRAF6 oligomerization. By performing SDD-AGE analysis with truncation mutants of ZDHHC11, we found that C-terminal region of ZDHHC11 (198-412 aa) could remarkably promoted TRAF6 oligomerization (Supplementary Figure 6). These results suggested that the interaction of ZDHHC11TRAF6 was important for ZDHHC11 to enhance TRAF6 oligomerization. In addition, we conducted immunostaining and observed more and larger granules of TRAF6 when ZDHHC11 and TRAF6 were cotransfected into HEK293T cells compared with TRAF6 transfected alone (Figure 5C). These data collectively demonstrate that ZDHHC11 enhanced TRAF6 oligomerization.

Given that TRAF6 oligomerization promotes its ubiquitin ligase activity, and that ZDHHC11 enhanced TRAF6 oligomerization, we next examined whether ZDHHC11 modulated TRAF6 E3 activity using an in vitro ubiquitination assay. First, we used the TRAF6-Ubc13/Uev2 system with E1 and wild-type ubiquitin and found that ZDHHC11 significantly increased synthesis of ubiquitination chains (Figure 5D). Because K63-linked polyubiquitination catalyzed by TRAF6 plays an important role in the initiation of TAK1 kinase activity (Wang et al., 2001), we next examined whether ZDHHC11 regulates the synthesis of K63-linked ubiquitination chains. As shown in Figure 5E, the synthesis of K63-linked ubiquitination chains was remarkably enhanced after addition of ZDHHC11 protein to the reaction mixture. Given that TRAF6 also mediates itself polyubiquitination, next we examined whether ZDHHC11 affected the ubiquitination of TRAF6. As shown in Figures 5F-I, ZDHHC11 overexpression increased wild-type and K63-lined ubiquitination of TRAF6 which conversely were reduced by ZDHHC11 knockdown. Given that oligomerization of TRAF6 induces TAK1 activation, next we performed in vitro TAK1 activation assay, and found that ZDHHC11 significantly enhanced TAK1 activation (Figure 5J). Collectively, these data suggest that ZDHHC11 enhances TRAF6 E3 activity by promoting TRAF6 oligomerization and E3 ligase activity, subsequently leading to TAK1 activation.

\section{DISCUSSION}

The NF-кB signaling plays an essential role in inflammation and innate immunity. Additionally, increasing evidence has demonstrated that the transcription factors of NF- $\mathrm{B}$ family are crucial for many steps in cancer initiation and progression ( $\mathrm{Li}$ and Verma, 2002; Taniguchi and Karin, 2018). Here, we found that ZDHHC11, a member of the DHHC palmitoyl transferase family,

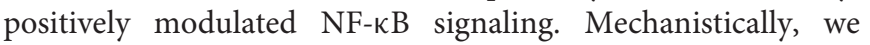
demonstrated that ZDHHC11 enhanced TRAF6 oligomerization, which augmented its E3 ligase activation.

ZDHHC11 is a member of the DHHC palmitoyl transferase family, which has been demonstrated to play important roles in regulating STING-mediated antiviral innate immune responses (Liu et al., 2018). Consistent with this study, we also found that ZDHHC11 positively modulated anti-DNA viral innate immune responses. Interestingly, $\mathrm{ZDHHC11}$ overexpression only activated the NF- $\kappa \mathrm{B}$, but not ISRE, promoter. Thus, we examined whether ZDHHC11 is involved in regulating activation of NF- $\mathrm{B}$ induced by other stimuli such as IL-1 $\beta$, TNF $\alpha$, and LPS. qRT-PCR showed that ZDHHC11 overexpression synergistically increased the level of IL- 8 mRNA induced by IL-1 $\beta$, but not $\mathrm{TNF} \alpha$, in HEK293 C6 cells. ZDHHC11 knockout showed that ZDHHC11 was involved in modulating NF- $\mathrm{B}$ activation induced by IL-1 $\beta$, LPS, and DNA virus infection in MEFs and macrophages. Furthermore, in vivo experiments showed that ZDHHC11 deficiency decreased IL-6 secretion in serum induced by LPS/D-galactosamine treatment and HSV-1 infection. Notably, the palmitoyl transferase activity of ZDHHC11 was not involved in NF-кB activation. Of note, we observed that mRNA levels of ZDHHC11 was not induced by the stimulation of IL- $1 \beta$, and IL- $\beta$ stimulation had no effect on the association between ZDHHC11 and TRAF6 (Supplementary Figure 7). It would be interesting to address the issue of what triggers the ZDHHC11-TRAF6 binding in the future study.

Tumor necrosis factor receptor-associated factor 6 acts as an E3 ubiquitin ligase together with Ubc13-Uev1A, which catalyzes K63-linked polyubiquitination and plays a critical role in NF- $\kappa \mathrm{B}$ signaling by regulating activation of the TAK1 and IKK complexes (Deng et al., 2000; Hu et al., 2017). TRAF6 oligomerization is critical for its E3 activity (Yin et al., 2009; Fu et al., 2018). In this study, Co-IP demonstrated that ZDHHC11 interacted with TRAF6 through the TRAF6-C domain that has been reported to function as a binding platform to regulate TRAF6 association with 


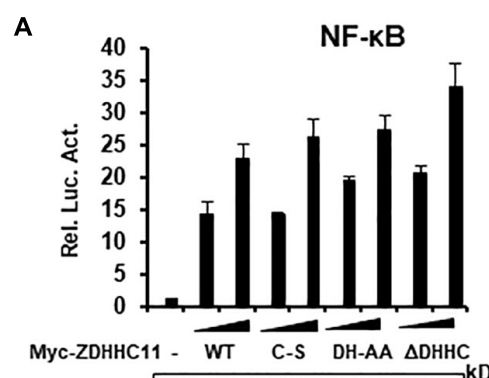

B Flag-TRAF6 - +++

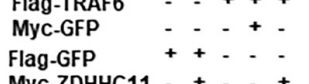

D Flag-TRAF6 + $6++$

Ub/E1/E2
Flag-ZDHHC11 +++++

Myc-ZDHHC11 $+\ldots+\ldots k \mathrm{kDa}$

SDD |IB: Flag

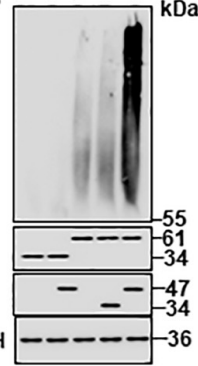

IB: Ub

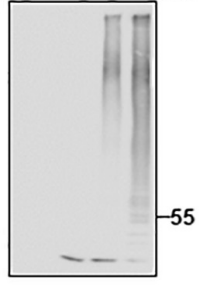

IB: Myc

IB: GAPDH

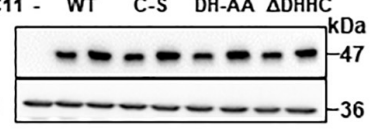

IB: Flag

IB: Myc

IB: GAPDH

IB: TRAF6

IB: Flag

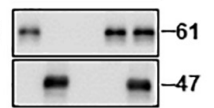

C
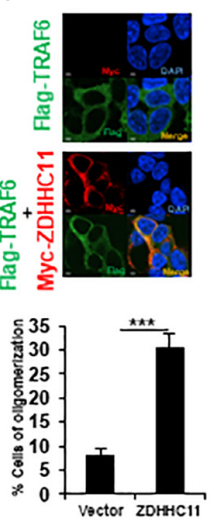

H

Flag-TRAF6

Myc-Ub

shZDHHC11-1

shZDHHC11-2
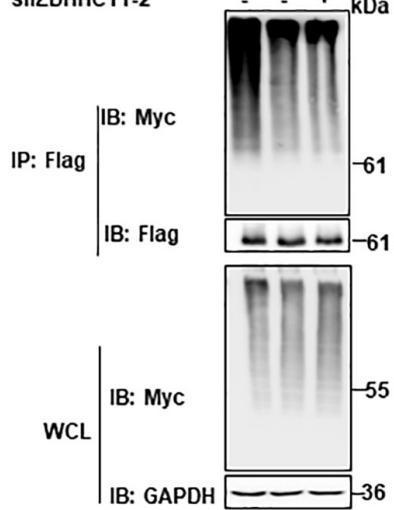

E

Flag-ZDHHC11

IB: Ub

IB: TRAF6

IB: Flag
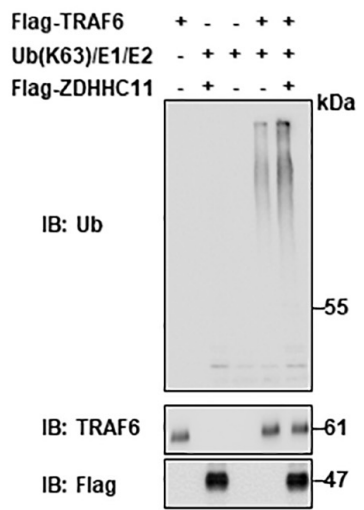

Flag-TRAF6 HA-Ub Myc-ZDHHC11

IB: HA IP: Flag IB: Fla

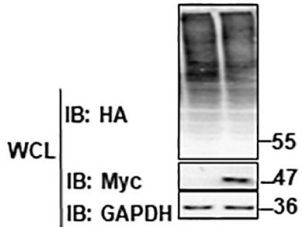

G
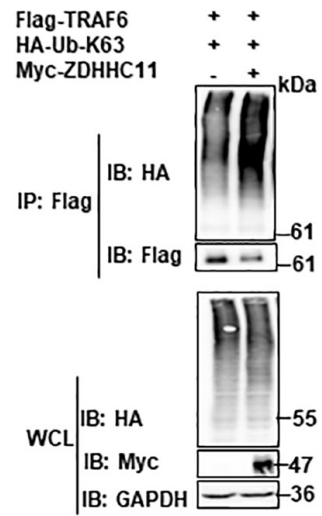

J

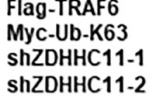

$++$

$+++$

$-+$

shZDHHC11-2

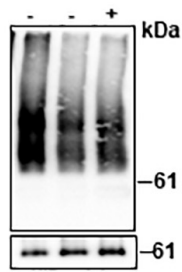

TAK1 complex ++++

Ub/E1/E2 - + + +

Flag-TRAF6 - -++

Flag-ZDHHC11 $-+++k \mathrm{kDa}$

IB: p-TAK1

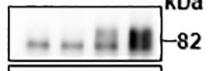

IB: TAK1
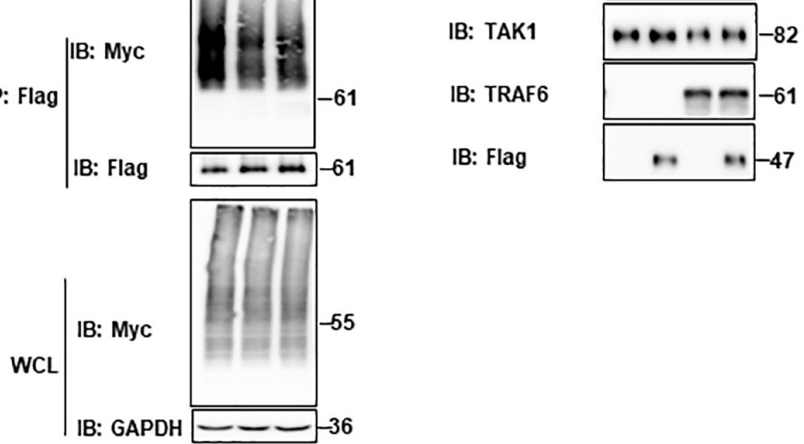

IB: Flag

IB: TRAF6

FIGURE 5 | ZDHHC11 increases TRAF6 E3 activity by enhancing TRAF6 oligomerization. (A) HEK293T cells were cotransfected with an empty vector or expression plasmids encoding wild-type ZDHHC11 (100 ng) or its mutants (100 ng) and a NF-kB-Luc reporter plasmid (20 ng). After 24 h, reporter assays (upper panel) and immunoblot assay (lower panels) were performed. (B) HEK293T cells were cotransfected with the indicated expression plasmids. $24 \mathrm{~h}$ later, cell lysates were separated by SDD-AGE (upper panel) or SDS-PAGE (lower panels), followed by immunoblot analysis. (C) HEK293T cells were transfected with the indicated plasmids (500 ng/each) for $24 \mathrm{~h}$, then stained with the indicated antibodies. The images were taken by confocal microscopy. Scale bars, $2 \mu \mathrm{m}$. The percentage of cells with larger granules was counted in 150 cells. (D,E) Purified TRAF6 and ZDHHC11 were incubated as indicated in a reaction mixture containing ATP, E1, E2, and $\mathrm{Ub}$ (D), or Ub-K63 (E) for $1 \mathrm{~h}$ at $30^{\circ} \mathrm{C}$, then analyzed by immunoblotting. (F,G) HEK293T cells were transfected with TRAF6 (1 $\left.\mu \mathrm{g}\right)$ and ZDHHC11 (1 $\left.\mu \mathrm{g}\right)$ or empty vector together with HA-tagged wild-type Ub (HA-Ub; F), HA-Ub-K63 (G) plasmids (1 $\mu$ g/each). At 24 h post-transfection, Co-IPs were performed with anti-Flag beads, followed by immunoblotting. (H,I) HEK293 C6 cells were infected with lentivirus expressing shRNA that targeted different coding regions of ZDHHC11 (shZDHHC11-1 and shZDHHC11-2) or an empty vector for $48 \mathrm{~h}$. Then cells were cotransfected with TRAF6 (1 $\mu \mathrm{g}$ ) and Myc-tagged wild-type Ub (Myc-Ub; H) or Myc-Ub-K63 (I) plasmids (1 $\mu \mathrm{g})$. After $24 \mathrm{~h}$ of transfection, cell lysates were harvested for immunoprecipitation with anti-Flag beads, followed by immunoblotting. (J) Purified TRAF6, ZDHHC11, and TAK1 complex were incubated as indicated in a reaction mixture containing ATP, E1, E2, and Ub for $1 \mathrm{~h}$ at $30^{\circ} \mathrm{C}$, then analyzed by immunoblotting. Data shown in (A) is representative of three independent experiments (mean $\pm \mathrm{SD}$ of duplicate experiments). ${ }^{\star \star *} \mathrm{P}<0.001$ significant versus the control groups. All blots are representative of three independent experiments. 
other proteins. ZDHHC11 overexpression significantly increased the level of IL8 mRNA induced by TRAF6 overexpression, whereas knockdown of TRAF6 significantly decreased NF$\kappa \mathrm{B}$ activation induced by ZDHHC11 overexpression. These results support the notion that ZDHHC11 targets TRAF6 to regulate NF- $\mathrm{B}$ signaling. Furthermore, we found that ZDHHC11 overexpression enhanced TRAF6 oligomerization, which subsequently increased E3 activity. These data suggest that ZDHHC11 positively modulates TRAF6 E3 activity by promoting its oligomerization.

Previous studies have indicated that ZDHHC11 and ZDHHC11B play critical roles in maintaining the oncogenic MYC-miR-150-MYB axis in Burkitt's lymphoma, and ZDHHC11 may be a biomarker to identify high-risk bladder cancer patients with disease progression (Yamamoto et al., 2007; Dzikiewicz-Krawczyk et al., 2017). In our study, ZDHHC11 overexpression activated NF- $\kappa \mathrm{B}$ signaling and it was involved in regulating $\mathrm{NF}-\kappa \mathrm{B}$ signaling induced by several stimuli such as Il-1 $\beta$, LPS, and a DNA virus. Because NF- $\kappa$ B signaling plays a crucial role in cancer initiation and progression, whether the function of ZDHHC11 in cancer development is related to its regulatory role in NF- $\mathrm{B}$ signaling needs to be investigated further.

In summary, we identified $\mathrm{ZDHHC11}$ as a positive modulator of NF- $\kappa$ B signaling. ZDHHC11 interacted with TRAF6 and promoted its oligomerization, which increased E3 activity. Our data provide a new insight into understanding the regulatory mechanism of TRAF6-mediated NF- $\kappa$ B signaling.

\section{MATERIALS AND METHODS}

\section{Ethics Statement}

All animal studies were performed in accordance with the recommendations in the Guide for the Care and Use of Laboratory Animals of the Ministry of Science and Technology of the People's Republic of China. The protocols for animal studies were approved by the Committee on the Ethics of Animal Experiments of the Institute of Zoology, Chinese Academy of Sciences (Approval number: IOZ15001).

\section{Cell Culture and Animals}

HEK293T and HeLa cells were bought from the Shanghai Cell Bank of Chinese Academy of Sciences (Shanghai, China). HEK293 C6 cells that ectopically express IL-1R were kindly provided by Dr. Zongping Xia in Zhengzhou University. Cells were cultured in Dulbecco's modified Eagle's medium (DMEM, Invitrogen) containing 10\% (v/v) fetal bovine serum (Invitrogen) and $1 \%$ streptomycin and penicillin. Zdhhcl1 $1^{+/+}$ and $Z d h h c 11^{-/-}$MEFs were isolated from 13.5-day-old embryos of $\mathrm{Zdhhc11^{+/+ }}$ and $Z d h h c 11^{-/-}$mice (The Jackson Laboratory). MEFs were cultured in complete DMEM containing $1 \mathrm{mM}$ sodium pyruvate, $10 \mu \mathrm{M}$ L-glutamine, $10 \mu \mathrm{M} \beta$ mercaptoethanol, and $1 \%$ non-essential amino acids. BMDMs were prepared as described previously (Li et al., 2013). Genomic DNA was extracted from 2-week-old mouse tails or cells for genotyping, followed by PCR analyses in accordance with the instructions from The Jackson Laboratory. The sequence of primers for genotyping is as follows:

$$
\begin{aligned}
& \text { \#1 (5'-3) CTGCCATACACCTAAATGCCTCAGC; } \\
& \text { \#2 (5'-3) TTTCGGAGCTGAAAAGCCAAGAAGG; } \\
& \text { \#3 (5'-3) ACTTGCTTTAAAAAACCTCCCACA; } \\
& \text { \#4 (5'-3) CCACATACCACACAGACATACACAGC. }
\end{aligned}
$$

\section{Plasmids}

Flag-tagged ZDHHC11, TRAF2, TRAF3, TRAF5, TRAF6, TAK1, IKK $\alpha$, IKK $\beta$, NEMO, P65 and; Myc-tagged ZDHHC11 were cloned into pcDNA3 or pEF vector. ZDHHC11 and TRAF6 mutants were generated by PCR using Pfu DNA polymerase. IFN- $\beta$, NF- $\kappa$ B, and ISRE luciferase reporter plasmids have been described previously (Zhao et al., 2012).

\section{Antibodies}

Rabbit anti-Flag was purchased from Sigma. Rabbit and mouse anti-Myc antibodies were purchased from MBL. Rabbit anti-pP65 (Ser536, 3033), anti-TAK1 (5206), anti-p-TAK1 (T184/187, 4508), anti-IKK $\alpha$ (61294), anti-IKK $\beta$ (8943), anti-p-IKK $\alpha / \beta$ (S176/180, 2697), and mouse anti-p-IкB $\alpha$ (Ser32/36, 9246) antibodies were from Cell Signaling Technology. Mouse anti-p65 (SC-8008), anti-TRAF2 (SC-876), anti-ubiquitin (SC-8017), and rabbit anti-IкB $\alpha$ (SC-371) were from Santa Cruz Biotechnology. Rabbit anti-TRAF6 (ab40675) was from Abcam. Mouse antiGAPDH (KM9002) and anti-Tubulin (KM9007) were from Sungene Biotechnology.

\section{Transfection and Luciferase Assay Reporter}

HEK293T cells were cotransfected with the indicated expression plasmids or an empty vector with a Renilla reporter plasmid and luciferase reporter plasmid that encoded IFN $\beta$-Luc, NF- $\kappa B-L u c$, or ISRE-Luc. The empty control plasmid was added to ensure that each transfection obtains the same amount of total DNA. $24 \mathrm{~h}$ after transfection, the cells were lysed for luciferase activity, and transfection efficiency was normalized to Renilla activity (Tao et al., 2020).

\section{Co-IP and Immunoblot Assay}

The Co-IP methods have been described previously (Zhao et al., 2012). Briefly, cells were lysed in lysis buffer with protease inhibitor cocktail (Roche) and incubated at $4^{\circ} \mathrm{C}$ with anti-Flag agarose beads (Sigma) or anti-Myc magnetic beads (Bimake) for $4 \mathrm{~h}$. The complexes were washed 3-4 times and subjected to immunoblot assay. For detecting multiple phosphoproteins, the cells were directly lysed in 1x SDS-PAGE sample loading buffer (50 mM Tris pH 6.8, 1\% mercaptoethanol, 2\% SDS, $0.01 \%$ bromophenol blue, and $10 \%$ glycerol). Immunoblotting was conducted using standard procedures.

\section{SDD-AGE Assay}

Cells were transfected as indicated and then lysed in lysis buffer (1\% NP40, $50 \mathrm{mM}$ Tris- $\mathrm{HCl}, 150 \mathrm{mM} \mathrm{NaCl}, 1 \mathrm{mM}$ EDTA, and $10 \%$ glycerol) with protease inhibitor cocktail (Roche) for $30 \mathrm{~min}$ at $4^{\circ} \mathrm{C}$. The cell lysates were centrifugated at $10,000 \mathrm{rpm}$ 
for $10 \mathrm{~min}$. The SDD-AGE assay was performed as described previously (Hou et al., 2011). Briefly, the supernatants were resuspended in $1 \mathrm{x}$ sample buffer $(0.5 \mathrm{x}$ TBE, $2 \%$ SDS, $10 \%$ glycerol, and $0.01 \%$ bromophenol blue), loaded on $1.5 \%$ agarose gel and electrophoresis was performed in the running buffer $(1 \mathrm{x}$ TBE and $0.1 \%$ SDS) with a constant voltage of $110 \mathrm{~V}$ for $45 \mathrm{~min}$ at $4^{\circ} \mathrm{C}$, followed by immunoblotting.

\section{Immunofluorescence}

HEK293T cells were cultured in gelatin-coated 12-well plates overnight, and then transfected with the indicated plasmids. After $24 \mathrm{~h}$, the cells were washed with phosphatebuffered saline, fixed with $4 \%$ paraformaldehyde for $10 \mathrm{~min}$, permeabilized with $0.2 \%$ Triton $\mathrm{X}-100$ for $15 \mathrm{~min}$, and then blocked with $5 \%(\mathrm{w} / \mathrm{v})$ bovine serum albumin for $30 \mathrm{~min}$, followed by incubating with primary and secondary antibodies. Imaging was performed under a Zeiss LSM 710 META laser scanning confocal system and ANDOR CR-DFLY505 confocal microscope equipped with a sCMOS Zyla 4.2 plus camera.

\section{In vitro Ubiquitination Assays}

HEK293T cells were transfected with Flag-ZDHHC11 or FlagTRAF6 and then cultured for $36 \mathrm{~h}$. The cells were lysed with lysis buffer (0.5\% NP40, 20 mM Tris-HCl pH7.5, $150 \mathrm{mM}$ $\mathrm{NaCl}, 10 \%$ glycerol, and $1 \mathrm{mM}$ EDTA) with protease inhibitor cocktail (Roche) and then purified with anti-Flag beads. The proteins were eluted by a Flag peptide after extensive washing with buffer (0.5\% NP40, 20 mM Tris- $\mathrm{HCl}$ pH 7.5, $500 \mathrm{mM} \mathrm{NaCl}$, $10 \%$ glycerol, and $1 \mathrm{mM}$ EDTA). In vitro ubiquitination assays were performed in a reaction mixture containing recombinant E1, Ubc13/Uev2, TRAF6, and ubiquitin (WT or K63-linked) in ATP buffer in the presence or absence ZDHHC11 protein. The reaction was incubated at $30^{\circ} \mathrm{C}$ for $1 \mathrm{~h}$ and terminated by addition of denaturing sample buffer, followed by $95^{\circ} \mathrm{C}$ heating for $5 \mathrm{~min}$. The samples were resolved on $6-18 \%$ or $10 \%$ SDS-PAGE gels, followed by immunoblotting with indicated antibodies.

\section{In vitro TAK1 Kinase Activation Assays}

HEK293T cells were infected with lentivirus expressing PCDHFlag-TAK1. At $100 \mathrm{~h}$ post-infection, the cells were lysed with lysis buffer (0.5\% Tritonx-100, 20 mM Tris-HCl pH7.5, $150 \mathrm{mM}$ $\mathrm{NaCl}, 10 \%$ glycerol, and $1 \mathrm{mM}$ EDTA) with protease inhibitor cocktail (Roche) and then purified with anti-Flag beads. The proteins were eluted by a Flag peptide after washing with buffer (0.5\% Tritonx-100, 20 mM Tris- $\mathrm{HCl} \mathrm{pH} \mathrm{7.5,} 150 \mathrm{mM} \mathrm{NaCl}$, $10 \%$ glycerol, and $1 \mathrm{mM}$ EDTA). TRAF6 and ZDHHC11 protein were purified as described in ubiquitination assays. In vitro TAK1 kinase activation assays were performed in a reaction mixture containing recombinant E1, Ubc13/Uev2, TRAF6, TAK1 complex and ubiquitin in ATP buffer with or without ZDHHC11 protein. The reaction was incubated at $30^{\circ} \mathrm{C}$ for $1 \mathrm{~h}$ and terminated by addition of denaturing sample buffer, followed by $95^{\circ} \mathrm{C}$ heating for $5 \mathrm{~min}$. The samples were resolved on $10 \%$ SDS-PAGE gels and then analyzed by immunoblotting with the indicated antibodies.

\section{Target Gene Knockdown by RNA Interference and Lentivirus-Mediated ShRNA}

HEK293T cells were transfected with siRNA that targeted TRAF6 or a non-targeting control (NC) at a final concentration of $30 \mathrm{nM}$ by the standard calcium phosphate transfection method. $24 \mathrm{~h}$ after transfection, the cells were transfected with the indicated plasmids using Lipofectamine 3000 (Invitrogen) for $24 \mathrm{~h}$ and then harvested for luciferase reporter assays and immunoblot analysis. The sequence for human TRAF6 siRNA were as follows $\left(5^{\prime}-3^{\prime}\right)$ : CUGUGCUGCAUCAAUGGCA.

HEK293 C6 and HeLa cells were infected with lentivirus that targeted two different regions of human ZDHHC11 (shZDHHC11-1 and shZDHHC11-2) or an empty vector for $48 \mathrm{~h}$. The cells were untreated or treated with IL-1 $\beta$ for the indicated times, followed by subsequent experiments. Knockdown efficiency was determined by qRT-PCR. The shRNA sequences against human $Z D H H C 11$ were as follows $\left(5^{\prime}-3^{\prime}\right)$ : shZDHHC11-1: CTCCAATGTCAGACTCATGAA; shZDHHC11-2: CCACCTTTGAGTAT CTCATTA.

\section{qRT-PCR}

Total RNA was isolated from cells with TRIZOL reagent (Invitrogen), cDNA was synthesized with a SuperScript III First-Strand cDNA Synthesis kit (Invitrogen). qRT-PCR was performed using SYBR Green Master Mix (Thermo Fisher) and Bio-Rad CFX connect system. Data were normalized to the abundance of GAPDH mRNA, and shown with the relative abundance of mRNA compared with the control group. The primers used were listed as follows $\left(5^{\prime}-3^{\prime}\right)$ :

hZDHHC11-S GGTGCAGACCCTGATAGTCG
hZDHHC11-AS GCACGTATGGATCTTTCCTCAC
hIL8-S ATAAAGACATACTCCAAACCTTTCCAC
hIL8-AS AAGCTTTACAATAATTTCTGTGTTGGC
hTNF $\alpha$-S CTGCCCCAATCCCTTTATT
hTNF - AS CCCAATTCTCTTTTTGAGCC
hIFIT1-S TACCTGGACAAGGTGGAGAA
hIFIT1-AS GTGAGGACATGTTGGCTAGA
hGAPDH-S ATGACATCAAGAAGGTGGTG
hGAPDH-AS CATACCAGGAAATGAGCTTG
mIl6-S TCGGAGGCTTAATTACACATGTTCT
mIl6-AS TGCCATTGCACAACTCTTTTCT
mTnf $\alpha-S$ TCCCCAAAGGGATGAGAAGTT
mTnf $\alpha-A S$ GTTTGCTACGACGTGGGCTAC
mGapdh-S AACTTTGGCATTGTGGAAGG
mGapdh-AS ACACATTGGGGGTAGGAACA
mIfnb1-S ATGGTGGTCCGAGCAGAGAT
mIfnb1-AS CCACCACTCATTCTGAGGCA
mIl-1 $\beta-S$ AAAGCCTCGTGCTGTCGGACC
mIl-1 $\beta-A S$ CAGGGTGGGTGTGCCGTCTT.

\section{In vivo LPS Treatment and Viral Infection and ELISA}

$Z d h h c 11^{+/+}$and $Z d h h c 11^{-/-}$mice were treated with LPS $(3 \mu \mathrm{g} / \mathrm{kg})$ and D-galactosamine $(250 \mathrm{mg} / \mathrm{kg})$ via intraperitoneal 
injection. Sera were collected at 1 and $3 \mathrm{~h}$ after injection to measure IL-6 by a mouse IL-6 ELISA kit (BD Biosciences) following with the manufacturer's instructions. Zdhhc11 $11^{+/+}$ and $Z d h h c 11^{-/-}$mice were infected with $H S V-1$ via tail vein injection at $2 \times 10^{7}$ plaque-forming units (PFU)/mouse. Sera were collected at 4 and $8 \mathrm{~h}$ after infection to measure IL-6 production by an ELISA kit.

\section{Statistical Analysis}

The data are presented as means \pm SD and two-tailed Student's $t$-test was used to examine significant differences between values under different experimental conditions. For all tests, $p$ values $<0.05$ were considered statistically significant.

\section{DATA AVAILABILITY STATEMENT}

The raw data supporting the conclusions of this article will be made available by the authors, without undue reservation.

\section{ETHICS STATEMENT}

The animal study was reviewed and approved by the Institute of Zoology, Chinese Academy of Sciences.

\section{AUTHOR CONTRIBUTIONS}

EL, DC, and QS designed the experiments and wrote the manuscript. EL, JS, JY, LL, QY, JZe, and JZh performed the experiments. EL and QS performed data analysis. All authors contributed to the article and approved the submitted version.

\section{REFERENCES}

Abe, T., and Barber, G. N. (2014). Cytosolic-DNA-mediated, STING-dependent proinflammatory gene induction necessitates canonical NF-kappaB activation through TBK1. J. Virol. 88, 5328-5341. doi: 10.1128/jvi.00037-14

Adhikari, A., Xu, M., and Chen, Z. J. (2007). Ubiquitin-mediated activation of TAK1 and IKK. Oncogene 26, 3214-3226. doi: 10.1038/sj.onc.1210413

Cao, Z., Xiong, J., Takeuchi, M., Kurama, T., and Goeddel, D. V. (1996). TRAF6 is a signal transducer for interleukin-1. Nature 383, 443-446. doi: 10.1038/ $383443 \mathrm{a} 0$

Daniotti, J. L., Pedro, M. P., and Valdez Taubas, J. (2017). The role of S-acylation in protein trafficking. Traffic 18, 699-710. doi: 10.1111/tra.12510

Deng, L., Wang, C., Spencer, E., Yang, L., Braun, A., You, J., et al. (2000). Activation of the IkappaB kinase complex by TRAF6 requires a dimeric ubiquitinconjugating enzyme complex and a unique polyubiquitin chain. Cell 103, 351-361. doi: 10.1016/s0092-8674(00)00126-4

Dzikiewicz-Krawczyk, A., Kok, K., Slezak-Prochazka, I., Robertus, J. L., Bruining, J., Tayari, M. M., et al. (2017). ZDHHC11 and ZDHHC11B are critical novel components of the oncogenic MYC-miR-150-MYB network in Burkitt lymphoma. Leukemia 31, 1470-1473. doi: 10.1038/leu.2017.94

Ea, C. K., Sun, L., Inoue, J., and Chen, Z. J. (2004). TIFA activates IkappaB kinase (IKK) by promoting oligomerization and ubiquitination of TRAF6. Proc. Natl. Acad. Sci. U. S. A. 101, 15318-15323. doi: 10.1073/pnas.040413 2101

\section{FUNDING}

This study was supported by Natural Science Foundation of China (Grant 31970895) and the Open Research Program of State Key Laboratory of Membrane Biology.

\section{ACKNOWLEDGMENTS}

We would like thank Zongping Xia (Zhengzhou University) for kindly providing HEK293 C6 cells and recombinant E1, Ubc13/Uev2 and ubiquitin protein.

\section{SUPPLEMENTARY MATERIAL}

The Supplementary Material for this article can be found online at: https://www.frontiersin.org/articles/10.3389/fcell.2021. 710967/full\#supplementary-material

Supplementary Figure 1 | Identification of $\mathrm{ZDHHC11}$ as a positive regulator in NF-к B signaling.

Supplementary Figure 2 | ZDHHC11 overexpression activates NF-кB downstream genes in HeLa cells.

Supplementary Figure 3 | ZDHHC11 knockdown reduces NF-kB activation stimulated by IL-1 $\beta$ in HeLa cells.

Supplementary Figure $4 \mid$ | Zdhhc11 deficiency reduces NF-кB activation stimulated by IL-1 $\beta$, LPS, and DNA virus infection.

Supplementary Figure 5 | TRAF6 interacts with ZDHHC11 through its TRAF C domain.

Supplementary Figure 6 | C-terminal (198-412aa) region of ZDHHC11 plays an important role in enhancing TRAF6 oligomerization.

Supplementary Figure $7 \mid \mathrm{IL}-1 \beta$ treatment has no effect on the mRNA level of ZDHHC11 and association with TRAF6.

Fu, T. M., Shen, C., Li, Q., Zhang, P., and Wu, H. (2018). Mechanism of ubiquitin transfer promoted by TRAF6. Proc. Natl. Acad. Sci. U. S. A. 115, 1783-1788. doi: $10.1073 /$ pnas.1721788115

Gorleku, O. A., Barns, A. M., Prescott, G. R., Greaves, J., and Chamberlain, L. H. (2011). Endoplasmic reticulum localization of DHHC palmitoyltransferases mediated by lysine-based sorting signals. J. Biol. Chem. 286, 39573-39584. doi: $10.1074 /$ jbc.m111.272369

Hopfner, K. P., and Hornung, V. (2020). Molecular mechanisms and cellular functions of cGAS-STING signalling. Nat. Rev. Mol. Cell Biol. 21, 501-521. doi: 10.1038/s41580-020-0244-x

Hou, F., Sun, L., Zheng, H., Skaug, B., Jiang, Q. X., and Chen, Z. J. (2011). MAVS forms functional prion-like aggregates to activate and propagate antiviral innate immune response. Cell 146, 448-461. doi: 10.1016/j.cell.2011.06.041

Hu, L., Xu, J., Xie, X., Zhou, Y., Tao, P., Li, H., et al. (2017). Oligomerization-primed coiled-coil domain interaction with Ubc13 confers processivity to TRAF6 ubiquitin ligase activity. Nat. Commun. 8:814.

Janssens, S., and Beyaert, R. (2002). A universal role for MyD88 in TLR/IL-1Rmediated signaling. Trends Biochem. Sci. 27, 474-482. doi: 10.1016/s09680004(02)02145-x

Kanayama, A., Seth, R. B., Sun, L., Ea, C. K., Hong, M., Shaito, A., et al. (2004). TAB2 and TAB3 activate the NF-kappaB pathway through binding to polyubiquitin chains. Mol. Cell 15, 535-548. doi: 10.1016/j.molcel.2004.08.008

Kang, J. U., Koo, S. H., Kwon, K. C., Park, J. W., and Kim, J. M. (2008). Gain at chromosomal region 5p15.33, containing TERT, is the most frequent genetic 
event in early stages of non-small cell lung cancer. Cancer Genet. Cytogenet. 182, 1-11. doi: 10.1016/j.cancergencyto.2007.12.004

Ko, P. J., and Dixon, S. J. (2018). Protein palmitoylation and cancer. EMBO Rep. 19:e46666.

Korycka, J., Lach, A., Heger, E., Boguslawska, D. M., Wolny, M., Toporkiewicz, M., et al. (2012). Human DHHC proteins: a spotlight on the hidden player of palmitoylation. Eur. J. Cell Biol. 91, 107-117. doi: 10.1016/j.ejcb.2011.09.013

Lemonidis, K., Werno, M. W., Greaves, J., Diez-Ardanuy, C., Sanchez-Perez, M. C., Salaun, C., et al. (2015). The zDHHC family of S-acyltransferases. Biochem. Soc. Trans. 43, 217-221. doi: 10.1042/bst20140270

Li, Q., and Verma, I. M. (2002). NF-kappaB regulation in the immune system. Nat. Rev. Immunol. 2, 725-734.

Li, X. D., Wu, J., Gao, D., Wang, H., Sun, L., and Chen, Z. J. (2013). Pivotal roles of cGAS-cGAMP signaling in antiviral defense and immune adjuvant effects. Science 341, 1390-1394. doi: 10.1126/science.1244040

Liu, S., Chen, J., Cai, X., Wu, J., Chen, X., Wu, Y. T., et al. (2013). MAVS recruits multiple ubiquitin $\mathrm{E} 3$ ligases to activate antiviral signaling cascades. Elife 2:e00785.

Liu, Y., Zhou, Q., Zhong, L., Lin, H., Hu, M. M., Zhou, Y., et al. (2018). ZDHHC11 modulates innate immune response to DNA virus by mediating MITA-IRF3 association. Cell. Mol. Immunol. 15, 907-916. doi: 10.1038/cmi.2017.146

Lomaga, M. A., Yeh, W. C., Sarosi, I., Duncan, G. S., Furlonger, C., Ho, A., et al. (1999). TRAF6 deficiency results in osteopetrosis and defective interleukin-1, CD40, and LPS signaling. Genes Dev. 13, 1015-1024. doi: 10.1101/gad.13.8. 1015

Mitchell, D. A., Vasudevan, A., Linder, M. E., and Deschenes, R. J. (2006). Protein palmitoylation by a family of DHHC protein S-acyltransferases. J. Lipid Res. 47, 1118-1127. doi: 10.1194/jlr.r600007-jlr200

Mulero, M. C., Huxford, T., and Ghosh, G. (2019). NF-kappaB, IkappaB, and IKK: integral Components of Immune System Signaling. Adv. Exp. Med. Biol. 1172, 207-226. doi: 10.1007/978-981-13-9367-9_10

Nakano, H., Oshima, H., Chung, W., Williams-Abbott, L., Ware, C. F., Yagita, H., et al. (1996). TRAF5, an activator of NF-kappaB and putative signal transducer for the lymphotoxin-beta receptor. J. Biol. Chem. 271, 14661-14664. doi: 10. 1074/jbc.271.25.14661

Narayanan, K. B., and Park, H. H. (2015). Toll/interleukin-1 receptor (TIR) domain-mediated cellular signaling pathways. Apoptosis 20, 196-209. doi: 10. 1007/s10495-014-1073-1

Park, H. H. (2018). Structure of TRAF Family: current Understanding of Receptor Recognition. Front. Immunol. 9:1999. doi: 10.3389/fimmu.2018.01999

Park, Y. C., Burkitt, V., Villa, A. R., Tong, L., and Wu, H. (1999). Structural basis for self-association and receptor recognition of human TRAF2. Nature 398, 533-538. doi: 10.1038/19110

Shi, J. H., and Sun, S. C. (2018). Tumor Necrosis Factor Receptor-Associated Factor Regulation of Nuclear Factor kappaB and Mitogen-Activated Protein Kinase Pathways. Front. Immunol. 9:1849. doi: 10.3389/fimmu.2018.01849

Taniguchi, K., and Karin, M. (2018). NF-kappaB, inflammation, immunity and cancer: coming of age. Nat. Rev. Immunol. 18, 309-324. doi: 10.1038/nri.2017. 142

Tao, X., Chu, B., Xin, D., Li, L., and Sun, Q. (2020). USP27X negatively regulates antiviral signaling by deubiquitinating RIG-I. PLoS Pathog. 16:e1008293. doi: 10.1371/journal.ppat.1008293

Verstrepen, L., Bekaert, T., Chau, T. L., Tavernier, J., Chariot, A., and Beyaert, R. (2008). TLR-4, IL-1R and TNF-R signaling to NF-kappaB: variations on a common theme. Cell. Mol. Life Sci. 65, 2964-2978. doi: 10.1007/s00018-0088064-8

Wang, C., Deng, L., Hong, M., Akkaraju, G. R., Inoue, J., and Chen, Z. J. (2001). TAK1 is a ubiquitin-dependent kinase of MKK and IKK. Nature 412, 346-351. doi: $10.1038 / 35085597$

Wu, C. J., Conze, D. B., Li, T., Srinivasula, S. M., and Ashwell, J. D. (2006). Sensing of Lys 63-linked polyubiquitination by NEMO is a key event in NF-kappaB activation [corrected]. Nat. Cell Biol. 8, 398-406. doi: 10.1038/ncb1384

Wu, J. F., Lee, C. H., Chen, H. L., Ni, Y. H., Hsu, H. Y., Sheu, J. C., et al. (2013). Copy-number variations in hepatoblastoma associate with unique clinical features. Hepatol. Int. 7, 208-214. doi: 10.1007/s12072-012-9350-y

Xie, P. (2013). TRAF molecules in cell signaling and in human diseases. J. Mol. Signal. 8:7. doi: 10.1186/1750-2187-8-7

Yamamoto, Y., Chochi, Y., Matsuyama, H., Eguchi, S., Kawauchi, S., Furuya, T., et al. (2007). Gain of 5p15.33 is associated with progression of bladder cancer. Oncology 72, 132-138.

Ye, H., Arron, J. R., Lamothe, B., Cirilli, M., Kobayashi, T., Shevde, N. K., et al. (2002). Distinct molecular mechanism for initiating TRAF6 signalling. Nature 418, 443-447. doi: 10.1038/nature00888

Yin, Q., Lin, S. C., Lamothe, B., Lu, M., Lo, Y. C., Hura, G., et al. (2009). E2 interaction and dimerization in the crystal structure of TRAF6. Nat. Struct. Mol. Biol. 16, 658-666. doi: 10.1038/nsmb.1605

Yoneyama, M., Kikuchi, M., Natsukawa, T., Shinobu, N., Imaizumi, T., Miyagishi, M., et al. (2004). The RNA helicase RIG-I has an essential function in doublestranded RNA-induced innate antiviral responses. Nat. Immunol. 5, 730-737. doi: $10.1038 /$ ni1087

Yount, J. S., Zhang, M. M., and Hang, H. C. (2013). Emerging roles for protein S-palmitoylation in immunity from chemical proteomics. Curr. Opin. Chem. Biol. 17, 27-33. doi: 10.1016/j.cbpa.2012.11.008

Zaballa, M. E., and van der Goot, F. G. (2018). The molecular era of protein S-acylation: spotlight on structure, mechanisms, and dynamics. Crit. Rev. Biochem. Mol. Biol. 53, 420-451. doi: 10.1080/10409238.2018.1488804

Zhao, Y., Sun, X., Nie, X., Sun, L., Tang, T. S., Chen, D., et al. (2012). COX5B regulates MAVS-mediated antiviral signaling through interaction with ATG5 and repressing ROS production. PLoS Pathog. 8:e1003086. doi: 10.1371/journal. ppat. 1003086

Conflict of Interest: The authors declare that the research was conducted in the absence of any commercial or financial relationships that could be construed as a potential conflict of interest.

Publisher's Note: All claims expressed in this article are solely those of the authors and do not necessarily represent those of their affiliated organizations, or those of the publisher, the editors and the reviewers. Any product that may be evaluated in this article, or claim that may be made by its manufacturer, is not guaranteed or endorsed by the publisher.

Copyright (c) 2021 Liu, Sun, Yang, Li, Yang, Zeng, Zhang, Chen and Sun. This is an open-access article distributed under the terms of the Creative Commons Attribution License (CC BY). The use, distribution or reproduction in other forums is permitted, provided the original author(s) and the copyright owner(s) are credited and that the original publication in this journal is cited, in accordance with accepted academic practice. No use, distribution or reproduction is permitted which does not comply with these terms. 